\title{
Evolution of chalcone isomerase from a non-catalytic ancestor
}

Miriam Kaltenbach $^{1 *}$, Jason R. Burke ${ }^{2 *}$, Mirco Dindo ${ }^{1,3}$, Anna Pabis ${ }^{4}$, Fabian S. Munsberg ${ }^{4}$, Avigayel Rabin ${ }^{1,5}$, Shina C.L. Kamerlin ${ }^{4}$, Joseph P. Noel ${ }^{2}$ and Dan S. Tawfik $^{1}$

${ }^{1}$ Department of Biological Chemistry, The Weizmann Institute of Science, Rehovot 76100, Israel

${ }^{2}$ Howard Hughes Medical Institute, Jack H. Skirball Center for Chemical Biology and Proteomics, The Salk Institute for Biological Studies, La Jolla, CA 92037, USA

${ }^{3}$ Current address: Department of Neuroscience, Biomedicine and Movement Sciences, Biological Chemistry Section, University of Verona, 37134 Verona, Italy

${ }^{4}$ Uppsala Biomedicinsk Centrum, Department of Cell and Molecular Biology, Uppsala University, Uppsala S-751 24, Sweden

${ }^{5}$ Current address: Department of Biological Chemistry, The Alexander Silberman Institute of Life Sciences, The Hebrew University of Jerusalem, Edmond J. Safra Campus, Jerusalem 91904, Israel

* These authors contributed equally to this work.

Corresponding authors:

Dan S. Tawfik

Department of Biological Chemistry

The Weizmann Institute of Science

Rehovot, 76100, Israel

Tel: +972-8-934-3637

Email: dan.tawfik@weizmann.ac.il

Joseph P. Noel

Howard Hughes Medical Institute

Jack H. Skirball Center for Chemical Biology and Proteomics

The Salk Institute for Biological Studies

La Jolla, CA 92037, USA

Tel:

Email: noel@salk.edu 


\section{Abstract}

2 The emergence of catalysis in a non-catalytic protein scaffold is a rare, unexplored

3 event. Chalcone isomerase $(\mathrm{CHI})$, a key enzyme in plant flavonoid biosynthesis, is

4 presumed to have evolved from a non-enzymatic ancestor related to the widely-

5 distributed fatty-acid binding proteins (FAPs) and a plant protein family with no

6 isomerase activity (CHILs for "CHI-like"). Ancestral inference confirmed that CHI

7 evolved from a protein lacking isomerase activity. We also identified four alternative

8 founder mutations, i.e. mutations that individually instated activity, including a

9 mutation that is not phylogenetically traceable. Despite strong epistasis in other cases

10 of protein evolution, CHI's laboratory reconstructed mutational trajectory shows

11 weak epistasis. Thus, enantioselective $\mathrm{CHI}$ activity can readily emerge despite a

12 catalytically inactive starting point. X-ray crystallography, NMR, and MD simulations

13 reveal reshaping of the active site toward a productive substrate-binding mode and

14 repositioning of the catalytic arginine that was inherited from the ancestral fatty-acid

15 binding proteins. 


\section{Introduction}

17 Enzymes generally diverge from other enzymes by exploiting preexisting promiscuous activities while maintaining key catalytic residues. Moreover, enzymes often evolve into regulatory or scaffolding proteins through loss of catalytic residues ${ }^{1}$. However, the opposite evolutionary trend seems exceedingly rare - emergence of enzyme catalysis starting from a non-catalytic, ancestral protein ${ }^{2-5}$. the biosynthesis of plant flavonoids - specialized metabolites involved in diverse biotic and abiotic functions including UV protection, flower color, pollen development, root nodulation, plant architecture and chemical defenses ${ }^{6}$. CHI catalyzes the enantioselective formation of the tricyclic flavanone $(S)$-naringenin from its bicyclic precursor chalconaringenin (Fig. 1a). However, the only two homologous protein families identified to date are non-enzymatic ${ }^{7}$ : the phylogenetically dispersed and presumably more ancient, fatty-acid binding proteins (FAP), and a family of closely related plant proteins dubbed CHI-like (CHIL). FAPs play a role in fatty-acid biosynthesis ${ }^{7}$ and are found in plants as well as in some algae, protists and bacteria. In contrast, $\mathrm{CHI}$ and CHIL have been identified only in plants. CHILs exhibit no CHI activity. Their expression correlates with flavonoid biosynthesis ${ }^{8,9}$, but their

34 biochemical activity remains unknown. Given that neither FAPs nor CHILs show CHI activity, and that FAPs are likely the oldest of the three families, the parsimonious hypothesis is that CHI evolved from a non-enzymatic protein ${ }^{7}$ However, given the evolutionary distance between FAPs and CHIs, and the rarity of cases where enzymes evolved from non-catalytic proteins, CHI's ancestor may have

39 been an isomerase, and activity was subsequently lost in CHILs. Phylogeny alone 
cannot reveal when $\mathrm{CHI}$ catalysis emerged, nor the trajectory that led from a nonenzymatic ancestor to a bona fide CHI. Therefore, we set out to address these questions using a combination of ancestral sequence inference, directed evolution, $\mathrm{x}$ ray crystallography, NMR, and MD simulations.

\section{Results}

\section{Ancestral sequence inference}

We inferred three ancestral nodes by maximum-likelihood: The most probable ancestor of all chalcone isomerases (ancCHI), of all CHI-like proteins (ancCHIL), and the $\mathrm{CHI} / \mathrm{CHIL}$ common ancestor (ancCC). Given the wide divergence between CHIs and FAPs, a very earlier ancestor was not inferred. Details of the procedure and prediction statistics are provided in Supplementary Results and Online Methods. Briefly, because protein sequence divergence between CHI, CHIL, and FAPs is high, and includes insertions and deletions (InDels), we generated a structure-based alignment (Supplementary Table 1, Supplementary Fig. 1, Supplementary Data Set 1). No systematic InDels were found between the CHI and CHIL lineages. Hence, the structural alignment was trimmed in loop regions and at the $\mathrm{N}$ - and $\mathrm{C}$-termini, and a phylogenetic tree was generated (Fig. 1b; see Supplementary Fig. 2 for the complete tree). Remaining gaps and ambiguously aligned positions were handled manually in the reconstructed ancestors (Supplementary Table 2, Supplementary Fig. 3). $\mathrm{CHIs}^{7,10}$ and, as expected, these were all present in the most probable ancCHI sequence (posterior probabilities $\mathrm{p}=0.84-0.98$ ). Of these, R34, T46, Y104 (ancCC 
63

sequence numbering is used throughout), which are highly conserved in extant CHIs (80-100\%, Supplementary Fig. 1, Supplementary Data Set 1), are present in ancCC $(\mathrm{p}=0.97-0.98)$. For the remaining two positions, different amino acids were predicted in ancCC vs. ancCHI (S111 vs. N and V188 vs. T). These residues were shown to be functionally less relevant ${ }^{10}$ and accordingly more variable (67-68\%) in extant CHIs, as reflected by lower posterior probabilities in ancCHI (0.84 and 0.91).

\section{The inferred CHI/CHIL ancestor exhibits no CHI activity}

The three inferred ancestors expressed in high yield as soluble proteins in E. coli and displayed high thermal stability (apparent midpoint melting temperatures $\left(\mathrm{T}_{\mathrm{m}}\right)>$ $75{ }^{\circ} \mathrm{C}$; Supplementary Table 3, Supplementary Fig. 4). AncCHI was an active CHI with a $k_{\text {cat }} / K_{M}$ of $2.1 \times 10^{5} \mathrm{M}^{-1} \mathrm{~s}^{-1}$ for $(S)$-naringenin formation, $\sim 40$-fold lower than that of Arabidopsis thaliana CHI (AtCHI, $7.7 \times 10^{6} \mathrm{M}^{-1} \mathrm{~s}^{-1}$, Fig. 2a; Supplementary Table 4). Like all characterized extant CHIs, ancCHI enantioselectively produces only $(S)$ naringenin (Fig. 2b; Supplementary Table 4). Isomerase activity above background was not detected either in ancCHIL (as in extant CHILs) or in ancCC (Fig. 2a, Supplementary Fig. 5). Nonetheless, as detailed below, the crystal structure of ancCC showed the characteristic CHI-CHIL fold. Therefore, ancCC appears to be correctly folded and stable, suggesting that the lack of enzymatic activity is not due to inference errors. However, we only reconstructed the statistically most probable sequence while in effect, ancestral inference yields a "cloud" of sequences that relate to the historical ancestor ${ }^{11-13}$. Results described below indicate that this "cloud" includes sequences that possess basal CHI activity, but the probability that ancCC's isomerase activity exceeded low, promiscuous levels is negligible. Therefore, our findings confirm the hypothesis that $\mathrm{CHI}$ evolved from a non-enzymatic protein. ${ }^{7}$ 

connects ancCC and ancCHI? We addressed this question using a phylogenetic that separate the inferred ancCC and ancCHI sequences. While these two sequences differ by 65 amino acids (out of 220), we posit that most of these non-conserved exchanges have no significant contribution to the acquisition of enzymatic activity. Therefore, to limit library size, we constructed a variant of ancCHI, dubbed ancCHI*, where exchanges in positions that are non-conserved or/and distant from the active site were excluded. AncCHI ${ }^{\star}$ differed from ancCC at 39 positions, possessed an identical structure (elaborated below), and exhibited a $k_{\text {cat }} / K_{M}$ of $1.0 \times 10^{5} \mathrm{M}^{-1} \mathrm{~s}^{-1}(\sim 2$ fold lower than ancCHI; Supplementary Fig. 6; Supplementary Table 4). The first library was generated by randomly incorporating 39 oligonucleotides into the ancCC gene, each encoding one amino acid exchange, at an average rate of 2 exchanges per

101 gene (Supplementary Tables 5-6). Expression in E. coli and activity-based screening of $>700$ clones in clarified cell lysates identified several variants with isomerization

103 rates above background (Fig. 2a). Several mutations were enriched, in particular 104 L108V (15 out of 23 clones, Supplementary Table 7a). A variant containing only 105 L108V was also identified ( 2.5-fold increased isomerization rate compared to the 106 uncatalyzed background reaction). The L108V variant, hereafter called ancR1, was 107 purified and its catalytic activity verified (Fig. 2b; Supplementary Tables 4 and 8). 108 The activity of this and other early mutants was too low to accurately determine $k_{\text {cat }}$ 109 and $K_{M}$ values, but assays with purified proteins consistently indicated rate 
110 enhancements above background in agreement with the lysate measurements

111 (Supplementary Tables 4 and 9).

113 ancestral mutations and shuffling of improved variants) and screening were

114 performed, yielding variant ancR7 that exhibited only 3-fold lower catalytic efficiency

115 compared to ancCHI ${ }^{*}\left(k_{\text {cat }} / K_{M}=7.6 \times 10^{4} \mathrm{M}^{-1} \mathrm{~s}^{-1}\right.$; Supplementary Tables 4, 6-8). All

116 variants showed the same enantioselectivity as extant CHIs (Fig. 2b; Supplementary

117 Table 4), suggesting that CHI's enantioselectivity was embedded in the inactive

118 ancCC scaffold. The emerging evolutionary trajectory represents a viable adaptive

119 pathway with gradual increases in activity and diminishing returns in later rounds

120 (Fig. 2c) ${ }^{14}$. Interestingly, ancR7 contained only 4 of the 5 previously identified

121 catalytic amino acids (S111N fixated, but not V188T) indicating that the V188T

122 exchange plays a relatively minor role in CHI's catalytic emergence.

123 CHI activity can arise independently via different non-epistatic founder mutations

124 Our initial screen identified L108V as the likeliest founder mutation, i.e. a single

125 mutation that can initiate CHI activity on its own. To identify alternative founder

126 mutations, we screened a second R1 library with a low mutation rate (0.2 amino acid

127 exchanges/gene) such that all possible single mutants were likely represented ( $>900$

128 clones, $\sim 5 \times$ oversampling, Supplementary Table 6). Two additional founder

129 mutations were identified (Fig. 1a): M36I, resulting in a 1.3-fold higher catalytic rate

130 compared to the background reaction, and I99L with a 2.0-fold increase. To explore

131 possible second mutations, we screened three low-mutation rate R2 libraries, each

132 based on one of the three founder mutations (Supplementary Table 6). The 
133 improved variants each contained the remaining founder mutations (Supplementary

134 Tables 10a-d). Indeed, the effect of these 3 founder mutations is largely additive since

135 all possible combinations of these mutations gave a smooth, 'uphill' trajectory (Fig.

136 3a). The absence of strong epistasis is further evidenced by the fact that reversion of

137 any of the three founder mutations in ancCHI${ }^{\star}$ resulted in only minor reductions in

$138 k_{\text {cat }} / K_{M}(4-6$-fold, Supplementary Table 9).

In addition to various combinations of founder mutations, the L108V library

140 contained improved variants with either K100T or S111N as the second mutation, the

141 latter being one of the two remaining active-site exchanges discussed above. A

142 combinatorial mutational analysis confirmed that neither of these second mutations

143 can serve as a founder mutation, as on their own they do not elicit activity in ancCC

144 (Fig. 3b). This analysis also explained why neither K100T nor S111N were found in

145 any selected variant: in the background of either M36I or I99L, K100T was nearly

146 neutral, while S111N reduced activity. That the effects of K100T and S111N develop

147 only in the presence of $\mathrm{L} 108 \mathrm{~V}$ reinforces the key role of this mutation in initiating

148 CHI's catalytic emergence.

By the fourth round, all three founder mutations, M36I, I99L, and L108V, and

150 both second-stage mutations, K100T and S111N, were fixed, and together, accounted

151 for most of the activity gains. The quintuple mutant is only 4 -fold less active in lysate

152 and 5-fold in terms of $k_{\text {cat }} / K_{M}$ compared to ancR4, which contains six additional

153 mutations. 
The above experiment sampled mutations that are phylogenetically traceable, i.e.,

157 sequence exchanges between the inferred ancestral nodes ancCC and ancCHI.

158 However, alternative routes may exist that were either not explored by natural

159 evolution or were historically explored, but are no longer visible in the phylogenetic

160 record. We thus subjected ancCC to random mutagenesis. We performed four rounds

161 of random mutagenesis by alternating between error-prone PCR (1.5 amino acid

162 substitutions/gene on average) and DNA shuffling, and screening for CHI activity

163 (Fig. 4a; Supplementary Tables 6, 11). Approximately 1100 clones were sampled in

164 round 1. Strikingly, none of the above-mentioned phylogenetic founder mutations

165 was observed. Most of the active variants identified were mutated at position F133 -

166 predominantly to Leu (7/19 sequenced clones) and also Ile or Ser (2 clones each).

167 Thus, F133L seems like an alternative to the phylogenetic trajectory, as far as this

168 trajectory can be inferred from the extant sequences. While there is a reasonable

169 probability that Leu was present in ancCC $(\mathrm{p}(\mathrm{L})=0.12$ compared to $\mathrm{p}(\mathrm{F})=0.84)$, it is

170 negligibly predicted in ancCHI $(p(F)=0.90, p(L)<0.01$, Supplementary Table 12).

171 Accordingly, position 133 is predominantly Phe in extant CHIs (85\%) while Leu is

172 rare (3.4\%) and Ile and Ser are not found (Supplementary Table 12). By round ep4,

173 higher activity evolved and four additional mutations fixated (Fig. 4a, Supplementary

174 Table 13), three of which resemble F133L in not being widely represented in extant

$175 \mathrm{CHI}$ and not exhibiting significant prediction probabilities in ancCHI

176 (Supplementary Table 12). Nonetheless, as observed in the ancestral trajectory, all

177 variants exclusively produced $(S)$-naringenin (Supplementary Table 4), reinforcing

178 our hypothesis that enantioselectivity was embedded in ancCC's pre-catalytic site. 
At first glance, it may seem like the error-prone trajectory, which reached a

181 ancestral trajectory (Fig. 2c). However, the two trajectories are not directly comparable. Firstly, the ancestral libraries primarily contained beneficial or neutral mutations, while the vast majority of random mutations are deleterious ${ }^{15}$. Secondly,

184 ancR4 contains 11 mutations compared to only five in epR4. However, the key 185 question is whether the two trajectories are bridgeable, or incompatible as commonly 186 observed in evolutionary trajectories (i.e., combining their mutations leads to loss of 187 activity $)^{16-18}$. To this end, we introduced the random founder mutation F133L into 188 various ancestral intermediates (Fig. 4b, Supplementary Table 9). F133L is slightly 189 deleterious in the background of the most catalytically active ancestral founder mutation (L108V) yet beneficial in the two others (M36I, I99L). When added to the 191 more advanced intermediates (R3, R7 and even ancCHI ${ }^{\star}$, F133L exerts no significant 192 effect, although it significantly reduces the activity of AtCHI. Thus, an alternative 193 trajectory based on F133L is in fact compatible with the phylogenetic trajectory. That 194 F133L is deleterious in an extant CHI indicates that incompatibility is the outcome of 195 the later stages of evolution, and also explains why Leu at position 133 is inferred with 196 negligible probability in CHI's ancestor. 
The structural and mechanistic basis of CHI's emergence

204 CHI's catalytic activity has been attributed to five residues ${ }^{7,10,19}$. R34 aligns the 205 substrate and stabilizes the transition-state by interacting with the attacking 2'206 hydroxyl (Fig. 1a, Fig. 5a) ${ }^{20}$. The other four residues form hydrogen bonds with the 207 carbonyl oxygen (T46, Y104) and 6'-OH (N111, T188; ancCC numbering) thus 208 directing substrate binding and favoring ring closure to give the (S)-enantiomer (Fig. 209 5a). R34, T46, and Y104 are most critical because their mutagenesis leads to dramatic 210 losses in activity (100-1000 fold reduction in $\left.k_{c a t}^{10,20}\right)$ and these three residues are 211 already present in ancCC (Fig. 5b). The catalytic arginine dates back to FAPs, where it 212 is absolutely conserved and coordinates the negatively charged carboxylate of the 213 bound fatty acids ${ }^{7}$. Why then is the inferred ancestor catalytically inactive, and how do 214 the identified mutations enable the emergence of catalysis and enantioselectivity? We solved the $\mathrm{x}$-ray crystal structures of various evolutionary intermediates

216 (Supplementary Table 14, Supplementary Fig. 7). The observed changes are subtle 217 and relate to the repositioning of specific active-site side chains rather than to global 218 rearrangements (Supplementary Table 15). Along the trajectory, mutations occurred 219 from the inside out, i.e. starting with first-shell mutations and progressively outward 220 from the active site to $2^{\text {nd }}$ and $3^{\text {rd }}$ shells (Supplementary Fig. 8). This trend suggests 221 that the late mutations fine-tune the structural effects of the early mutations ${ }^{14}$. Most noticeably, substitution of L108 by the shorter Val side chain in ancR1 223 enlarges the active-site cavity thus favoring productive substrate binding modes (Fig. 224 5c). When crystals of ancCC (1.5 $\AA$ resolution) and ancR1 (1.4 $\AA$ ) were soaked with 225 racemic $(R / S)$-naringenin (Fig. 5b, Supplementary Fig. 7), we observed a ligand 
227 PDB ID 1EYQ, Fig. 5a) ${ }^{19}$ and likely is non-productive. Because complexes could not

228 be obtained for the other variants, we performed MD simulations to further explore 229 the two contrasting binding modes (dubbed "ancCC-like" and "CHI-like").

230 Specifically, we computationally placed chalconaringenin in either the productive or 231 non-productive orientation into the crystal structures of various evolutionary 232 intermediates, and followed their positional changes over time (Fig. 5d, 233 Supplementary Figs. 9-10). When the substrate was placed in the CHI-like mode, it 234 remained in this orientation in all structures, supporting that this mode is indeed the 235 productive one (Fig. 5d). However, when the substrate was initially placed in the non236 productive ancCC-like mode, a complex trend was observed. In both ancCC and 237 ancR1, the substrate tends to either dissociate or remain in the active site while 238 adopting a variety of alternative non-productive orientations (Fig. 5d, 239 Supplementary Fig. 11). In ancR3, a more advanced evolutionary intermediate, 240 productive binding was first observed and in ancR7, non-productive ancCC-like 241 binding was eliminated (Fig. 5d). Control simulations of the ancCC and ancR1 crystal 242 structures in complex with $(S)$-naringenin showed that the product complexes were 243 stable over the course of the simulation, indicating that the observed instabilities of 244 the ancCC-like substrate binding mode are not a simulation artifact (Supplementary 245 Fig. 12). Thus, one important factor in the emergence of CHI activity is a change in 246 substrate positioning.

247 Concomitantly, changes in the conformational ensemble of the catalytic 248 arginine occurred. R34 assumes various rotamers in the structures of different 249 evolutionary intermediates, and also in extant CHIs (overall, the position of the 250 guanidinium carbon varies by up to $7.7 \AA$; Fig. 6a). Nonetheless, it appears that a 
251 consistent change in the most populated rotamers accompanied the emergence of

252 isomerase activity, as indicated by both NMR and MD simulations. The NMR

253 chemical shift of the guanidinium $\mathrm{N}^{\varepsilon}$ is well resolved. Three other arginines that reside

254 on the protein surface were mutated to lysines (R113K, R115K, R134K) in key

255 evolutionary intermediates (ancCC-, ancR1-, ancR3- and ancR7- $\Delta \mathrm{R}$; see Online

256 Methods). This resulted in an unambiguous resonance signature for the catalytic

257 arginine side chain in the ${ }^{15} \mathrm{~N}-{ }^{1} \mathrm{H}$ HSQC spectra of the studied variants

258 (Supplementary Fig. 13). Aggregation of ancR3- $\Delta \mathrm{R}$ excluded it from our NMR

259 analyses, but changes in the steady-state heteronuclear NOE effect were readily

260 measured in the other variants, providing dynamics of pico- to nanosecond thermal

261 fluctuations of ${ }^{15} \mathrm{~N}-{ }^{1} \mathrm{H}$ bond vectors ${ }^{21}$. The NOE intensity for $\mathrm{R} 34$ is low relative to

262 average backbone values in ancCC-, ancR1- and ancR7- $\Delta \mathrm{R}$, suggesting that the

263 guanidinium moiety of R34 displays faster motion than the proteins' overall tumbling

264 (Fig. 6b). The decrease in the relative NOE intensity of R34 between ancCC- $\Delta \mathrm{R}$ and

265 ancR1- $\Delta \mathrm{R}$ is consistent with an increase in $\mathrm{R} 34$ side chain mobility in ancR1- $\Delta \mathrm{R}$. This

266 increased flexibility may be attributable to increased available space in the active site

267 due to the L108V substitution (Fig. 5c). The relative NOE intensity increases again in

268 ancR7- $\Delta \mathrm{R}$, suggesting that R34 becomes less flexible in this advanced evolutionary

269 intermediate. Foremost, a large change in chemical shift was observed, indicating a

270 change in R34's average chemical environment (Fig. 6c). The modulation in mobility

271 and average location of R34 was recapitulated in MD simulations that included ancR3

272 (Fig. 6d). A significant narrowing in the ensemble of accessible arginine rotamers was

273 observed along the evolutionary trajectory, with ancR1's ensemble being most

274 dispersed (Fig. 6d). The increased mobility of R34 in ancR1 compared to both the 
275 starting point, ancCC, and the advanced intermediates, ancR3 and ancR7, was also

276 reflected in the root-mean square fluctuation (RMSF) of its side chain

277 (Supplementary Fig. 14). We conclude that the emergence of CHI catalysis occurred

278 via reshaping of the active-site cavity to allow the substrate to bind in a productive

279 mode, thereby also repositioning R34 and enabling it to exert its catalytic effect ${ }^{20}$.

\section{Discussion}

282 Our results indicate that CHI evolved from a catalytically inactive ancestor thus

283 showing that emergence of catalysis and stereospecificity in non-catalytic scaffolds is a

284 feasible evolutionary scenario. That said, a bacterial $\mathrm{CHI}^{22}$ has evolved independently

285 in an enzymatic protein fold, exemplifying that plant $\mathrm{CHI}$ is an exception rather than

286 the rule. The actual, historical ancestor may have exhibited some isomerase activity,

287 but this activity is unlikely to have exceeded a low, promiscuous level. The three

288 founder mutations that initiate $\mathrm{CHI}$ activity were individually inferred in ancCC with

289 tangible probabilities $(\mathrm{p}(\mathrm{I} 36)=0.11, \mathrm{p}(\mathrm{L} 99)=0.14, \mathrm{p}(\mathrm{L} 108)=0.04)$. However, the

290 likelihood that all three were present, giving rise to a relatively active enzyme (Fig. 3A;

291 specific activity $\left.\sim 6 \times 10^{3} \mu \mathrm{mol} / \mathrm{min} / \mathrm{mg}\right)$, is negligible $(\mathrm{p}<0.001)$.

That the inferred ancestor is devoid of $\mathrm{CHI}$ activity is also interesting because

293 other inferred enzyme ancestors were found to be multi-functional, typically

294 harboring varying levels of the activities found in the extant protein families that

295 diverged from them ${ }^{23}$. Here, we have obtained an ancestor that is not multi-

296 functional, at least with respect to the isomerase activity. However, what the function

297 of this ancestor was remains unknown, foremost because the function of

298 contemporary CHILs is also unknown. Presumably, the function of ancCC or extant 
299 CHILs relates to binding of phenylpropanoids as suggested by binding of $(S)$ 300 naringenin to ancCC in a mode that differs from CHI's.

301 Unraveling the ancestral function is a challenge also because at the time when 302 ancCC and the early CHI precursors emerged, flavonoid biosynthesis must have been 303 at its infancy ${ }^{24}$. This ancestral context is obviously lost in modern plants. Nonetheless, 304 in the context of an ancient, rudimentary metabolism, emergence from a nonenzymatic protein, and via intermediates that are relatively inefficient enzymes as 306 shown here, seems plausible. Given the high spontaneous rate of chalcone cyclization 307 (half-life for isomerization $\sim 3.6 \mathrm{~h}$ ), a weak catalyst or even a binding protein that 308 directs enantioselectivity could prove beneficial to the fitness of the organismal 309 host $^{25,26}$.

CHI's evolution seems unique not only in having its origins in an inactive

311 ancestor, but also in following a smooth, largely additive trajectory. In fact, we 312 surmise that these two features, and also the relatively facile spontaneous rate of 313 chalcone cyclization, are related; namely, it is the absence of strong epistasis and the 314 pre-existing uncatalyzed cyclization rate that made ancCC a feasible evolutionary 315 starting point despite its lack of initial activity. Multiple parallel trajectories to CHI 316 activity exist - four different founder mutations were identified and various additive 317 combinations thereof (Fig. 3 and 4). This trend is in contrast to previous studies 318 supporting the prevalence of epistasis in protein evolution, including sign epistasis, 319 and especially between founder mutations ${ }^{14,16,27-33}$. Beyond enzymatic function, the 320 mutations that initiated CHI's function affected stability - the apparent melting 321 temperatures of the evolutionary intermediates were reduced by up to $13{ }^{\circ} \mathrm{C}$ 322 compared to ancCC (Supplementary Table 3). Given the high stability of ancCC ${ }^{34}$, 
323 this destabilization had no phenotypic effects, but the actual, historical intermediates

324 may have been affected. Nevertheless, in terms of function, the mutations along CHI's

325 emergence show uncharacteristically additive effects. This may relate to the

326 preexistence of three key catalytic residues; however, key catalytic residues also

327 preexisted in enzyme trajectories that showed strong epistasis ${ }^{14,16,35,36}$. Low energetic

328 and steric demands of the reaction may be another factor, but these are also not

329 unique to this trajectory ${ }^{14,16}$. A more likely explanation in our view is that the

330 combined likelihood of emergence from a completely inactive starting point and via a

331 rugged, epistatic trajectory is exceedingly low. In other words, for an enzymatically

332 inactive protein to be a viable starting point, founder mutations must arise with

333 relatively high likelihood.

334 Finally, that CHI's ancestor is inactive despite all key catalytic residues being

335 in place, analogous to the current limitations of de novo enzyme design: That catalytic

336 residues can be placed within a suitable cavity, yet without taking into account

337 dynamics, resulting in geometries that only roughly match catalytically productive

338 conformations, yields poor enzymes at best which generally need to be improved by

339 directed evolution ${ }^{37-39}$. The presence of catalytic residues is necessary, but not

340 sufficient. However, once an initial activity level arise, mutations in other residues,

341 often distal from the active site, enable large improvements in catalytic efficiency ${ }^{40,41}$.

342 As seen in the evolution of CHI, subtle changes in substrate positioning and in the

343 position and flexibility of the catalytic residues relative to the bound substrate can

344 trigger activity in an inactive pocket. 
347 We thank Kesava-Phaneendra Cherukuri for help with the synthesis of

348 chalconaringenin, Brendan Duggan and Xuemei Huang for assistance with NMR,

349 Gordon Louie for assistance with protein x-ray data collection and processing, and

350 George Cortina for help with analyzing the simulations. This work was funded by the

351 Israel Science Foundation Grant 980/14 and the Sasson \& Marjorie Peress

352 Philanthropic Fund (D.S.T.); the United States National Science Foundation grant

353 EEC-0813570 (J.P.N.); the Knut and Alice Wallenberg Foundation, Wenner-Gren

354 Foundations and the European Research Council (S.C.L.K.). Computer time was

355 provided by the Swedish National Infrastructure for Computing. J.P.N. is the Arthur

356 and Julie Woodrow Chair and a Howard Hughes Medical Institute investigator. D.S.T.

357 is the Nella and Leon Benoziyo Professor of Biochemistry.

\section{Author Contributions}

360 M.K. performed ancestral inference with assistance from A.R. M.K. performed

361 directed evolution. M.K., M.D., and J.R.B. performed mutagenesis, protein expression,

362 stable isotope labeling, and biochemical characterization of the proteins. J.R.B., M.K.,

363 D.S.T. and J.P.N. performed and analyzed protein x-ray crystallography and NMR.

364 A.P. and S.C.L.K. performed and analyzed MD simulations with assistance from 365 F.S.M. D.S.T. and J.P.N. planned and directed the project, and, together with M.K.,

366 J.R.B., A.P., and S.C.L.K., designed the experiments. M.K., J.R.B., J.P.N. and D.S.T.

367 wrote and edited the manuscript. 
bioRxiv preprint doi: https://doi.org/10.1101/174128; this version posted August 10, 2017. The copyright holder for this preprint (which was

not certified by peer review) is the author/funder, who has granted bioRxiv a license to display the preprint in perpetuity. It is made available under aCC-BY-NC-ND 4.0 International license.

370 The authors declare no competing financial interests.

371 


\section{Online Methods}

\section{Ancestral reconstruction}

374 To generate a phylogenetic tree, we mined the $1 \mathrm{KP}$ transcriptome database ${ }^{42}$ for $\mathrm{CHI}$

375 and CHIL sequences. We also included sequences of several biochemically

376 characterized $\mathrm{CHI}$ proteins as well as sequences from ncbi protein BLAST. We

377 covered all major plant lineages (liverworts, mosses, lycophytes, ferns, gymnosperms,

378 and angiosperms) except for hornworts where no CHI or CHIL sequences could be

379 identified among the three transcriptomes (and zero genomes) available at the time.

380 While mosses contain CHILs, no CHI sequences were found in this lineage. Due to

381 the large number of available transcriptomes, redundant sequences $(\geq 70 \%)$ were

382 removed. However, because the early taxa (liverworts, mosses) were underrepresented

383 in the databases, we included several such sequences despite higher identity. Overall,

$38488 \mathrm{CHI}$ and $43 \mathrm{CHIL}$ sequences were included (Supplementary Table 1). Six

385 previously reported $\mathrm{FAPb}$ sequences that are the most closely related to $\mathrm{CHI}$ and

$386 \mathrm{CHIL}^{7}$ were included as outgroup. A structure-based alignment (Supplementary Fig.

387 1, Supplementary Data Set 1) was created with Expresso ${ }^{43}$ using the crystal structures

388 of AtCHI (PDB ID 4DOI, TAIR gene accession no. At3g55120), CHIL (4DOK,

389 At5g05270), and FAPb (4DOL, At1g53520). Poorly aligned regions (loops, N- and C-

390 termini) were trimmed. Positions that could not be trimmed unambiguously were

391 initially kept. A phylogenetic tree was generated with MrBayes $^{44}$ and showed a

392 consensus from a 1 million generation run (Fig. 1b; Supplementary Fig. 2). The tree

393 was largely consistent with the tree of life. Note that moss CHILs take up the outgroup

394 position within the CHIL clade. However, the relationship between basal land plants 
395 is still under debate ${ }^{45}$. AncCHI, ancCHIL, and ancCC were predicted using FastML

396 and the JTT substitution matrix ${ }^{46}$. Alternative substitution models were tested and

397 gave nearly identical results. By default, we chose the amino acids with the highest 398 posterior probabilities to derive the most probable ancestral sequences. Remaining 399 gaps and ambiguously aligned positions were handled manually as described in detail 400 in Supplementary Fig. 3 and Supplementary Table 2. The N- and C-terminal amino 401 acids were predicted with large ambiguity and hence, identical adaptor sequences 402 derived from AtCHI were added to each ancestor (Supplementary Fig. 1,

403 Supplementary Table 2). Ultimately, we obtained three 220-amino acid proteins, 404 with pairwise sequence identities of $89 \%$ for ancCC/ancCHIL, $70 \%$ for 405 ancCC/ancCHI, and $60 \%$ for ancCHI/ancCHIL.

\section{Protein expression and purification}

407 Synthetic genes for the three ancestors were ordered from Hylabs. All variants were 408 cloned into pHis8 (a modified pET28 vector containing an N-terminal His8-tag) 409 using Nco I and Hind III. Plasmids were transformed into E. coli BL21 (DE3). Cells 410 were grown at $37^{\circ} \mathrm{C}$ in $\mathrm{LB}$ containing $50 \mu \mathrm{g} / \mathrm{mL}$ Kanamycin to an $\mathrm{OD}_{\mathrm{OD}} \mathrm{D}_{60 \mathrm{~nm}}$ of $0.6-1$.

411 Overnight expression was induced by addition of 1M IPTG and the temperature 412 reduced to $18{ }^{\circ} \mathrm{C}$. Cells were harvested by centrifugation, resuspended and lysed by 413 sonication in Column Washing buffer (50 mM Tris- $\mathrm{HCl}, \mathrm{pH}$ 8.0, $500 \mathrm{mM} \mathrm{NaCl}, 20$ $414 \mathrm{mM}$ imidazole, $10 \%$ glycerol) supplemented with $1 \%$ Tween $20,10 \mathrm{mM} \beta$ 415 mercaptoethanol, $100 \mu \mathrm{g} / \mathrm{mL}$ Lysozyme, benzonase (Novagen) at a dilution of $10^{4}-10^{5}$, 416 and protease inhibitor cocktail for purification of His-tagged proteins (Sigma). Cell 417 debris was removed by centrifugation and the clarified lysate passed through a $45 \mu \mathrm{M}$ 418 filter prior to purification with Nickel beads (Adar Biotech). The Elution buffer 
419 differed from the Column Washing buffer in that it contained $250 \mathrm{mM}$ imidazole.

420 Proteins were dialyzed overnight against Activity buffer (50 mM HEPES pH 7.5, 150

$421 \mathrm{mM} \mathrm{NaCl}$ ) and concentrated over spin-column concentrators if necessary. Protein

422 concentration was determined using the Pierce BCA Protein Assay Kit (Thermo

423 Fisher Scientific).

\section{Synthesis of chalconaringenin}

425 Chalconaringenin was synthesized as previously described ${ }^{47}$ with minor modifications. 426 Naringenin (2 g, Sigma-Aldrich) was treated with $5 \% \mathrm{NaOH}$ in methanol $(50 \mathrm{~mL})$ 427 under reflux for 2 hours. Subsequently, $200 \mathrm{~mL}$ of $1 \mathrm{M} \mathrm{HCl}$ were added to the mixture 428 and the aqueous layer was extracted three times with ethyl acetate. The combined 429 organic layers were washed with brine and water, dried over $\mathrm{Na}_{2} \mathrm{SO}_{4}$, and evaporated. 430 The crude product was further purified by silica gel column chromatography using a 431 hexane:ethylacetate gradient (100\% hexane, then 7:1, 3:1, 2:1). ${ }^{1} \mathrm{H}$ NMR (400 MHz, 432 DMSO- $\left.d_{6}\right): \delta 12.56\left(\mathrm{~s}, \mathrm{OH}-2^{\prime} / 6^{\prime}\right), 10.46$ (s, OH-4'), 10.12 (s, OH-4), 7.95 (d, J=15.57 $433 \mathrm{~Hz}, \mathrm{H}-\beta), 7.64(\mathrm{~d}, J=15.57 \mathrm{~Hz}, \mathrm{H}-\alpha), 7.51$ (d, J=8.32 Hz, H-2/6), 6.81 (d, J=8.32 Hz, H$4343 / 5), 5.83\left(\mathrm{~s}, \mathrm{H}-3^{\prime} / 5^{\prime}\right) ;{ }^{13} \mathrm{C}$ NMR (100 MHz, DMSO- $\left.d_{6}\right): \delta 191.88,164.86,164.54,159.96$, $435 \quad 142.49,130.50,126.26,123.96,116.14,104.39,95.03$.

\section{Enzyme assays}

437 Naringenin formation was followed by the decrease in absorbance at $390 \mathrm{~nm}$ 438 (chalconaringenin is yellow; naringenin is colorless), typically in a Synergy HT 439 spectrophotometer (Bio-TEK). To determine the activity in crude cell lysates, cells 440 were grown at a $500 \mu \mathrm{L}$ culture volume in 96-well plates and lysed as described below 441 under Screening. Lysates were diluted (from no dilution up to a 10,000-fold dilution 
442 for extant CHIs) to determine initial rates of chalconaringenin isomerization (in the

443 range of $\mu \mathrm{M} / \mathrm{min})$. One volume of $200 \mu \mathrm{M}$ chalconaringenin in $50 \mathrm{mM}$ HEPES, $\mathrm{pH}$

4447.5 and $5 \%$ ethanol was added to one volume of diluted cell lysate. Initial rates were

445 normalized to cell density $\left(\mathrm{OD}_{600 \mathrm{~nm}}\right)$, corrected for the dilution factor, and averaged.

446 For the data shown in Fig.s 2-4 and Tables S4 and 9, each variant was grown in at

447 least two wells and the average determined. The whole experiment (cell growth,

448 protein expression, lysis and enzymatic assay) was repeated three times and the

449 combined average determined. For directed evolution, each well contained a different

450 variant (except for the respective control, i.e. the best variant from the previous round,

451 which was grown in at least triplicate on each plate). Improved variants were regrown

452 and assayed in triplicate.

453 Specific activity was determined with purified proteins ( $\mu$ mol product generated per

454 min per mg protein) at a substrate concentration of $100 \mu \mathrm{M}$. For determination of

455 kinetic parameters, assays were performed at 12 different substrate concentrations

$456(4.5-180 \mu \mathrm{M})$ in $50 \mathrm{mM}$ HEPES $\mathrm{pH} 7.5$ with $5 \%$ ethanol as co-solvent. The

457 spontaneous, background rates in buffer were subtracted, and the net initial rates were

458 directly fit to the Michaelis-Menten model using Kaleidagraph. In cases where no

459 significant rate saturation was observed, $k_{\text {cat }} / K_{M}$ was extracted by a linear fit. The rate

460 constant of the spontaneous reaction was calculated from a linear fit of initial rates in

461 buffer $\left(k_{\text {uncat }}=1.05 \times 10^{-3} \mathrm{~s}^{-1}\right.$, Supplementary Fig. 5). The spontaneous isomerisation

462 rate of chalconaringenin is relatively high. Hence, for the early evolutionary

463 intermediates, assays were performed at enzyme concentration that exceeded

464 substrate concentration $\left([\mathrm{E}]_{0}>[\mathrm{S}]_{0}\right)$. These could reveal $\mathrm{k}_{\text {cat }}$ values that are lower than 
$465 \mathrm{k}_{\text {uncat. }}$ For those proteins reported as inactive (e.g. ancCC) no activity above the

466 background rate was detected at up to $50 \mu \mathrm{M}$ protein.

\section{Determination of enantioselectivity}

468 Proteins were incubated with $60 \mu \mathrm{M}$ chalconaringenin in $50 \mathrm{mM}$ HEPES pH 7.5 for

46930 minutes at room temperature. For low-activity mutants, up to $1 \mathrm{mM}$ of protein

470 (>10-fold excess over substrate) was used. Naringenin was extracted with 0.5 volumes

471 of ethyl acetate. The organic phase was concentrated in a SpeedVac, centrifuged to

472 remove residual precipitated protein, and the sample analyzed on a chiral HPLC

473 column (Lux 5u Cellulose-4, $150 \times 4.6 \mathrm{~mm}$, Phenomenex) using a linear gradient

$474(88 \%$ hexane $/ 12 \%$ ethanol, $0.1 \%$ TFA $)$ and a flow rate of $1.5 \mathrm{ml} / \mathrm{min}$.

475 Chalconaringenin (retention time $5.6 \mathrm{~min}$ ), R-naringenin $(8.1 \mathrm{~min}$ ) and S-naringenin

476 (6.7 $\mathrm{min})$ were detected by absorbance at $310 \mathrm{~nm}$, where substrate and products

477 absorb to a similar extent. The traces of $(R)$-naringenin detected in case of the low-

478 activity variants are likely due to the spontaneous background reaction that generates

479 racemic product.

480 Thermostability assays

481 Solutions of $10 \mu \mathrm{M}$ protein were heated with 10x SYPRO Orange (Sigma) in $50 \mathrm{mM}$

482 HEPES pH 7.5 to a final temperature of 25 up to $95^{\circ} \mathrm{C}$, at a ramp of $0.9^{\circ} \mathrm{C} / \mathrm{min}$, in a

483 Viia 7 Real-Time PCR system (Applied Biosystems). The increase in fluorescence that

484 likely results from protein unfolding was monitored (excitation $488 \mathrm{~nm}$, emission

$485500-750 \mathrm{~nm})$. The apparent melting temperature $\left(\mathrm{T}_{\mathrm{m}}\right)$ is the midpoint of the resulting

486 temperature-fluorescence transition curve and was determined from the minimum

487 value of the first derivative. Measurements were performed in triplicate and average 
$488 \mathrm{~T}_{\mathrm{m}}$ values determined. The stability of selected variants was also measured by

489 following changes in tryptophan fluorescence upon heat-induced unfolding in a

490 Prometheus NT.48 NanoDSF instrument (NanoTemper Technologies). Solutions of

$49110 \mu \mathrm{M}$ protein were heated in $50 \mathrm{mM}$ HEPES $\mathrm{pH} 7.5$ from 20 to $95^{\circ} \mathrm{C}$ at a ramp of

$4922{ }^{\circ} \mathrm{C} / \mathrm{min}$, the observed fluorescence ratios at (excitation $280 \mathrm{~nm}$; emission

$493330 \mathrm{~nm} / 350 \mathrm{~nm}$ ) were plotted against temperature, and $T_{\mathrm{m}}$ values determined as the

494 minimum of the first derivative.

495 Cloning and library construction

496 Phylogenetic libraries

497 To reduce library size, we sought to remove neutral mutations. To this end, an 498 evolutionary conservation analysis was performed with ConSurf ${ }^{48}$ (Supplementary

499 Fig. 6a). Considering also the location of each substitution in the protein structure

500 (using the crystal structure of Medicago sativa CHI with bound product, PDB ID

501 1eyq), we identified and reverted 29 non-conserved and/or peripheral substitutions in 502 ancCHI to ancCC's amino acids, thus yielding ancCHI ${ }^{\star}$ (Supplementary Fig. 6b).

503 The catalytic activity of ancCHI ${ }^{*}$ was confirmed as similar to ancCHI $(\sim 2$-fold lower

$504 k_{c a t} / K_{M}$, Supplementary Table 4). The libraries were created with ISOR (Incorporating

505 Synthetic Oligonucleotides via Gene Reassembly ${ }^{49}$ ) at an average mutation rate of 2

506 amino acid exchanges/gene. For the round 1 library, ancCC was shuffled with 39

507 oligonucleotides, each containing one mutation to ancCHI's amino acid

508 (Supplementary Table 5). In cases where the amino acid exchange could only have

509 occurred by two base substitutions, the oligonucleotide was partially randomized to

510 include the plausible intermediary amino acid(s). Because several of the ancestral 
511 exchanges were at nearby positions, the primer set was updated after each round to

512 avoid the reversion of ancCHI mutations that were fixated in the previous rounds (see

513 Supplementary Table 5 for an example). Overall, seven rounds of library

514 construction and screening were performed. Low-mutation rate libraries were

515 generated in the same manner, but at a mutation rate of 0.2 amino acid

516 exchanges/gene. To ensure that all single mutations were represented with significant

517 probability, $\sim 930$ variants were screened for each of the four low-rate libraries $(\sim 5 \times$

518 oversampling). More details on library generation and screening are given in

519 Supplementary Table 6.

520 Random mutagenesis libraries

521 Random libraries were created by alternating rounds of error-prone PCR and DNA

522 shuffling. Error-prone PCR was performed using the GeneMorph II Random

523 Mutagenesis kit (Agilent) according to the manufacturer's instructions. The average

524 mutation rate was determined by sequencing a sample of randomly selected variants,

525 and libraries with an average of 1.2 amino acid substitutions/gene ( 2 nucleotide

526 substitutions/gene) were screened. DNA shuffling was performed with StEP

527 (Staggered Extension Process) $)^{50}$. More details on library generation and screening are

528 given in Supplementary Table 6.

529 Site-directed mutagenesis

530 Mutants were constructed as described in the QuikChange Site-Directed Mutagenesis

531 manual (Agilent). 
Library screening

534 Libraries were clones into pHis8, transformed into E. coli BL21 (DE3) and plated on

535 kanamycin LB-agar plates. Single colonies were picked into deep 96-well plates and

536 grown overnight at $30{ }^{\circ} \mathrm{C}$ in $200 \mu \mathrm{L} \mathrm{LB}$ per well supplemented with $50 \mu \mathrm{g} / \mathrm{mL}$

537 kanamycin. Wells containing fresh LB/Kanamycin $(500 \mu \mathrm{L})$ were inoculated with 25

$538 \mu \mathrm{L}$ of this preculture and protein expression was induced as above. Cells pelleted by

539 centrifugation and the supernatant removed. Cell lysis was achieved by freezing for 30

540 minutes at $-80{ }^{\circ} \mathrm{C}$, resuspension in $200 \mu \mathrm{L}$ of Activity buffer supplemented with $0.1 \%$

541 Triton-X100, $100 \mu \mathrm{g} / \mathrm{mL}$ Lysozyme, and 0.25-2.5 U/mL benzonase (Novagen) and

542 incubation for 30 minutes at room temperature. Cell debris was pelleted by

543 centrifugation and $20-100 \mu \mathrm{L}$ of clarified lysate (or dilutions thereof, depending on the

544 activity level of the library) were removed to assay for CHI activity. Substrate buffer

545 (Activity buffer supplemented with 1\% DMSO and chalconaringenin) was added to a

546 final volume of $200 \mu \mathrm{L}$ at a final substrate concentration of $50-180 \mu \mathrm{M}$, and the initial

547 rates of naringenin formation were determined. Improved variants (compared to the

548 best variant of the previous round) were picked and re-grown in triplicate, initial rates

549 re-measured, and the average values determined. Plasmid DNA was isolated and

550 variants were sequenced (Supplementary Tables 7, 10 and 11). The plasmids were

551 retransformed, and the resulting single colonies were used to inoculate new cultures

552 for activity determination and protein purification.

\section{X-ray crystallography}

554 Proteins purified by Ni-NTA affinity chromatography were prepared for 555 crystallization by elution from a Superdex 200 size exclusion column in buffer 

ancR1 grew overnight at a concentration of $150 \mathrm{mg} / \mathrm{ml}$ in $42 \%$ MPD and $50 \mathrm{mM}$ acetic acid (pH 4.5). Crystals were then transferred to a condition with 65\% MPD, 50 $\mathrm{mM}$ acetic acid $(\mathrm{pH} 4.5), 20 \mathrm{mM}$ naringenin and soaked for three days. Protein

561 crystals of ancR2 were grown overnight at a concentration of $100 \mathrm{mgs} / \mathrm{ml}$ in $25 \%$ PEG 4K, 2.5 M ammonium formate and $100 \mathrm{mM}$ succinic acid (pH 5.5). Protein crystals of ancR5 were grown over 2 weeks at a concentration of $140 \mathrm{mgs} / \mathrm{ml}$ in $3.5 \mathrm{mM}$

564 ammonium sulfate and 100mM TAPS (pH 8.5). Protein crystals of ancR7 were grew 565 over one month at a concentration of $50 \mathrm{mgs} / \mathrm{ml}$ in $500 \mathrm{mM} \mathrm{NaCl}, 100 \mathrm{mM}$ NaCitrate 566 tribasic dihydrate ( $\mathrm{pH} 5.6$ ), and 2\% ethylene imine polymer. Protein crystals of ancR3 567 formed overnight at a concentration of $200 \mathrm{mg} / \mathrm{ml}$ in 25\% PEG 4K, $2.5 \mathrm{M}$ ammonium 568 formate and $100 \mathrm{mM}$ succinic acid, $\mathrm{pH}$ 5.5. Protein crystals of ancCHI ${ }^{\star}$ grew over one 569 week at a concentration of $100 \mathrm{mg} / \mathrm{ml}$ in $4.5 \mathrm{M} \mathrm{NaCl}$ and acetic acid, $\mathrm{pH}$ 4.5. Protein 570 crystals of epR4 grew in two days at a concentration of $120 \mathrm{mg} / \mathrm{ml}$ in $29 \%$ PEG 4K, 2.5

$571 \mathrm{M}$ ammonium formate and $50 \mathrm{mM}$ succinic acid, $\mathrm{pH}$ 5.5. Protein crystals were frozen 572 in liquid nitrogen and data were collected at $100 \mathrm{~K}$ at beamlines 8.2.1 and 8.2.2 at the 573 Advanced Light Source, Lawrence Berkeley National Laboratory. Data were integrated 574 with iMOSFLM ${ }^{51}$ and scaled with SCALA ${ }^{52}$. Arabidopsis thaliana CHI (AtCHI, PDB: 575 4DOI) was used as a search model for molecular replacement ${ }^{7}$, and phases were solved 576 with PHASER ${ }^{53}$. Models were modified in COOT $^{54}$ and refined with PHENIX ${ }^{55}$. 577 Statistics for data collection and refinement are in Supplementary Table 14. 578 Coordinates and structure factors have been deposited in the Protein Data Bank 579 under accession codes 5WKR (ancCC), 5WKS (ancR1), 5WL3 (ancR2), 5WL4 
bioRxiv preprint doi: https://doi.org/10.1101/174128; this version posted August 10, 2017. The copyright holder for this preprint (which was

not certified by peer review) is the author/funder, who has granted bioRxiv a license to display the preprint in perpetuity. It is made available under aCC-BY-NC-ND 4.0 International license.

(ancR3), 5WL5 (ancR5), 5WL6 (ancR7), 5WL7 (ancCHI*), and 5WL8 (epR4).

Constructs of ancCC, ancR1 and ancR7 were generated with the substitutions: R115K,

R117K, and R136K for the purpose of unambiguously resolving the ${ }^{1} \mathrm{H}^{\varepsilon}-{ }^{15} \mathrm{~N}^{\varepsilon}$ arginine signal of R34. The specific activity of ancR1- $\Delta \mathrm{R}$ was essentially the same compared to ancR1 (1.7-fold reduction). While the specific activity of ancR7- $\Delta \mathrm{R}$ was 15 -fold lower

586 than that of its parent ancR7, it is still a highly active enzyme (Supplementary Table 9). AncCC- $\Delta \mathrm{R}$, like ancCC, shows no activity, but is well-folded as shown by the NMR data and therefore likely functional. Proteins were expressed using ${ }^{15} \mathrm{~N}$

589 isotopically-labeled ammonium sulfate in minimal media, and purified by affinity 590 chromatography followed by removal of the His8-tag and finally ion exchange 591 chromatography. The protein samples were then prepared at a final concentration of $5921 \mathrm{mM}$ in a buffer composed of $50 \mathrm{mM} \mathrm{NaPO}_{4}, 5 \mathrm{mM}$ dithiothreitol, and $10 \% \mathrm{D}_{2} \mathrm{O}(\mathrm{pH}$ 593 6.1). ${ }^{1} \mathrm{H}-{ }^{15} \mathrm{~N}$ HSQC spectra were measured at $298 \mathrm{~K}$ on an $800 \mathrm{MHz}$ Bruker AVANCE

594 NEO solution state NMR spectrometer, equipped with a $5 \mathrm{~mm}$ cryogenically cooled

595 TCI probe. ${ }^{15} \mathrm{~N}-\left\{{ }^{1} \mathrm{H}\right\} \mathrm{NOEs}$ were measured with presaturated and unsaturated 596 experiments and recorded interleaved with a relaxation delay of 5.5 seconds. ${ }^{15} \mathrm{~N}-$ $597\left\{{ }^{1} \mathrm{H}\right\}$ NOE values were calculated from the ratio of peak intensities from the 598 presaturated to the unsaturated experiments. Experiments were performed in 599 triplicate to estimate error based on standard deviation between the calculated ${ }^{15} \mathrm{~N}-$ $600\left\{{ }^{1} \mathrm{H}\right\} \mathrm{NOE}$ value for each separate run. Unassigned amide backbone peaks downfield of $6018.5 \mathrm{ppm}$ represent structured residues in the protein and were selected to calculate the 602 average heteronuclear NOE of backbone values for comparison with the R34 603 sidechain. ${ }^{15} \mathrm{~N}$ longitudinal (T1) relaxation experiments were performed at $800 \mathrm{MHz}$ 
604 with 2 duplicate points for error estimation. Experiments were collected with T1

605 delays of 30, 300, 600(x2), 900, 1200(x2), 1800, 2400, and 3600ms. Peak intensities

606 were measured in Sparky ${ }^{56}$ and fitting of the exponential decay curves was performed

607 using the RelaxGUI ${ }^{57}$. Calculated T1 values for the $\mathrm{R} 34{ }^{1} \mathrm{H}^{\varepsilon}-{ }^{15} \mathrm{~N}^{\varepsilon}$ resonance are $1.137 \pm$

$6080.029 \mathrm{sec}, 1.154 \pm 0.029 \mathrm{sec}$, and $0.9691 \pm 0.035 \mathrm{sec}$ for $\operatorname{ancCC}(3 \mathrm{~K})$, ancR1(3K) and

609 ancR7(3K), respectively. Data were processed using NMRpipe ${ }^{58}$, Sparky ${ }^{56}$ and Relax ${ }^{57}$.

\section{MD Simulations}

611 Simulations of both the substrate-free enzymes, as well as substrate-bound complexes

612 were performed on ancCC, ancR1, ancR3, ancR7, and additionally on ancCHI and

$613 A t \mathrm{CHI}$ for the enzyme-substrate complexes, starting from the coordinates provided in

614 the respective PDB IDs listed under "X-ray crystallography" and 4DOI for AtCHI".

615 Only Chain A was retained for the simulations, and all heteroatoms and water

616 molecules further than $4 \AA$ away from the protein were removed. Missing N- and C-

617 terminal residues were modeled with Modeller v. $9.18^{59}$, using the standard presets,

618 and selecting coordinates based on the lowest $\mathrm{z}$-score. The ionization states of all

619 titratable residues, and the protonation states of histidine side chains, was determined

620 by empirical screening with $\mathrm{PROPKA}^{60}$ and histidine protonation patterns were

621 assigned based on visual examination of the local environment of each side chain. The

622 substrate was placed manually in the active site by analogy with $M s C H I$ (PDB ID

$6231 \mathrm{EYQ}^{19}$ ) for the productive (CHI type II like) binding mode, and the ancR1 structure

624 for the non-productive binding mode, respectively. The only exception to this was

625 ancCC, where a structure with the product in the non-productive binding mode was

626 available to use as a template. In addition to the simulations of the substrate-bound

627 complexes, simulations of ancCC and ancR1 variants in complex with the product 
were performed. These simulation were based on the crystallographic coordinates of

629 the two systems with the product bound (PDB IDs 5WKR and 5WKS), and were

630 performed in order to validate that the simulation protocol can reproduce the stability

631 of the product complex found in the non-productive binding mode in those crystal

632 structures. The model systems for these simulations were prepared using the same

633 procedure as described above for the simulations of substrate-free

634 enzymes and substrate-bound complexes.

635 All simulations were performed using the AMBER16 simulation package ${ }^{61}$ using the 636 AMBER ff14SB force field ${ }^{62}$ and the General Amber Force Field (GAFF) ${ }^{63}$ for the 637 protein and substrate/product, respectively. Partial charges for chalconaringenin were 638 calculated at the HF/6-31G* level of theory, using the Gaussian09 simulation 639 package $^{64}$, and fitted using the standard restrained electrostatic potential (RESP) 640 procedure $^{65}$ (Supplementary Table 16). All starting structures for the simulations 641 were prepared using the LEaP module of AMBER. The structures were solvated in a 642 truncated octahedron of TIP3P ${ }^{66}$ water molecules, extending to at least $10 \AA$ from the 643 protein atoms. $\mathrm{Na}^{+}$and $\mathrm{Cl}^{-}$ions were added to obtain a charge neutralized system with 644 an ion concentration of $0.15 \mathrm{M}$.

645 All systems were initially minimized (1000 steps steepest descent followed by 646 conjugate gradient minimization) with a $25 \mathrm{kcal} \mathrm{mol}^{-1} \AA^{-2}$ harmonic positional 647 restraint applied to all solute atoms, followed by two more minimization steps with 648 the restraint dropped to $5 \mathrm{kcal} \mathrm{mol}^{-1} \AA^{-2}$. The minimized systems were then gradually 649 heated from 100 to $300 \mathrm{~K}$ over 40 ps simulation time at constant volume (NVT 650 ensemble), with the temperature equilibrated for a further $60 \mathrm{ps}$. The density of the 651 system was then equilibrated by performing 300 ps of constant pressure (NPT) 
652 simulations with isotropic position scaling, using the Berendsen barostat ${ }^{67}$ for

653 pressure control. The $5 \mathrm{kcal} \mathrm{mol}^{-1} \AA^{-2}$ positional restraint was retained for both heating

654 and density equilibration, and this restraint was gradually released over 350 ps of

655 NVT simulations, with the restraint completely removed for a final 50 ps of

656 equilibration time. An additional $50 \mathrm{kcal} \mathrm{mol}^{-1} \mathrm{rad}^{2}$ harmonic restraint was applied to

657 the C1-C2-C3-C4 torsion angle of the substrate (see Supplementary Table 16 for

658 atom numbering) to keep this torsion angle within the range of $-20^{\circ}$ to $90^{\circ}$; this

659 restraint was also removed in the last 50 ps of the initial equilibration. Finally, the

660 initially equilibrated systems were subjected to $100 \mathrm{~ns}$ of fully unrestrained production

661 simulations in an NVT ensemble. This protocol was repeated five times for each

662 system to generate five independent trajectories, resulting in $500 \mathrm{~ns}$ of simulation time

663 per system, the RMSD convergence of which is shown in Supplementary Fig. 15. A 2

664 fs time step was used throughout, and all bonds involving hydrogen atoms were

665 constrained using the SHAKE algorithm ${ }^{68}$. The temperature was controlled using

666 Langevin dynamics ${ }^{69}$ with a $1 \mathrm{ps}^{-1}$ collision frequency, and long-range electrostatic

667 interactions were calculated using the Particle Mesh Ewald (PME) method $^{70}$. An $8 \AA$

668 cutoff was used to describe non-bonded interactions.

669 Finally, all simulations were analyzed using CPPTRAJ from the AmberTools 670 package $^{61}$, Visual Molecular Dynamic package ${ }^{71}$ and MDTraj 1.8.0 library ${ }^{72}$, and the

671 structure graphics were prepared using UCSF Chimera package ${ }^{73}$. 
674 1. Adrain, C. \& Freeman, M., New lives for old: evolution of pseudoenzyme

675 function illustrated by iRhoms. Nat Rev Mol Cell Biol 13, 489-498 (2012).

676 2. Ortmayer, M. et al., An oxidative N-demethylase reveals PAS transition from 677 ubiquitous sensor to enzyme. Nature 539, 593-597 (2016).

678 3. Taga, M.E. et al., BluB cannibalizes flavin to form the lower ligand of vitamin 679 B12. Nature 446, 449-453 (2007).

680 4. Tam, R. \& Saier, M.H., Jr., A bacterial periplasmic receptor homologue with 681 catalytic activity: cyclohexadienyl dehydratase of Pseudomonas aeruginosa is 682 homologous to receptors specific for polar amino acids. Res Microbiol 144, 165-169 683 (1993).

684 5. Yuhara, K. et al., Enzymatic characterization and gene identification of 685 aconitate isomerase, an enzyme involved in assimilation of trans-aconitic acid, from 686 Pseudomonas sp. WU-0701. FEBS J 282, 4257-4267 (2015).

687 6. Koes, R.E., Quattrocchio, F., \& Mol, J.N.M., The Flavonoid Biosynthetic 688 Pathway in Plants: Function and Evolution. BioEssays 16, 123-132 (1994).

689 7. Ngaki, M.N. et al., Evolution of the chalcone-isomerase fold from fatty-acid 690 binding to stereospecific catalysis. Nature 485, 530-533 (2012).

691 8. Morita, Y. et al., A chalcone isomerase-like protein enhances flavonoid 692 production and flower pigmentation. Plant J 78, 294-304 (2014).

693 9. Jiang, W. et al., Role of a chalcone isomerase-like protein in flavonoid 694 biosynthesis in Arabidopsis thaliana. J Exp Bot 66, 7165-7179 (2015).

695 10. Jez, J.M., Bowman, M.E., \& Noel, J.P., Role of hydrogen bonds in the reaction 696 mechanism of chalcone isomerase. Biochemistry 41, 5168-5176 (2002). 
697 11. Bar-Rogovsky, H. et al., Assessing the prediction fidelity of ancestral

698 reconstruction by a library approach. Protein Eng Des Sel 28, 507-518 (2015).

699 12. Eick, G.N. et al., Robustness of Reconstructed Ancestral Protein Functions to 700 Statistical Uncertainty. Mol Biol Evol 34, 247-261 (2017).

701 13. Randall, R.N. et al., An experimental phylogeny to benchmark ancestral 702 sequence reconstruction. Nat Commun 7, 12847 (2016).

703 14. Tokuriki, N. et al., Diminishing returns and tradeoffs constrain the laboratory 704 optimization of an enzyme. Nat Commun 3, 1257 (2012).

705 15. Tokuriki, N. et al., The stability effects of protein mutations appear to be 706 universally distributed. J Mol Biol 369, 1318-1332 (2007).

707 16. Salverda, M.L. et al., Initial mutations direct alternative pathways of protein 708 evolution. PLoS Genet 7, e1001321 (2011).

709 17. Kaltenbach, M. et al., Reverse evolution leads to genotypic incompatibility 710 despite functional and active site convergence. Elife 4 (2015).

711 18. Dickinson, B.C. et al., Experimental interrogation of the path dependence and 712 stochasticity of protein evolution using phage-assisted continuous evolution. Proc 713 Natl Acad Sci U S A 110, 9007-9012 (2013).

714 19. Jez, J.M., Bowman, M.E., Dixon, R.A., \& Noel, J.P., Structure and mechanism 715 of the evolutionarily unique plant enzyme chalcone isomerase. Nat Struct Biol 7, 786$716791(2000)$.

717 20. Burke, J.R. et al., Origin of Enantioselective Catalysis by a Guanidine Moiety 718 in Chalcone Isomerases. submitted manuscript (2017). 
21. Farrow, N.A. et al., Backbone dynamics of a free and phosphopeptidecomplexed Src homology 2 domain studied by 15N NMR relaxation. Biochemistry 33,

721 5984-6003 (1994).

722

22. Thomsen, M. et al., Structure and catalytic mechanism of the evolutionarily unique bacterial chalcone isomerase. Acta Crystallogr D Biol Crystallogr 71, 907-917

724 (2015).

23. Gumulya, Y. \& Gillam, E.M., Exploring the past and the future of protein evolution with ancestral sequence reconstruction: the 'retro' approach to protein engineering. Biochem J 474, 1-19 (2017).

24. Weng, J.K. \& Chapple, C., The origin and evolution of lignin biosynthesis.

729 New Phytol 187, 273-285 (2010).

25. Bar-Even, A. \& Salah Tawfik, D., Engineering specialized metabolic pathways-

731 -is there a room for enzyme improvements? Curr Opin Biotechnol 24, 310-319 (2013).

732 26. Keller, M.A., Piedrafita, G., \& Ralser, M., The widespread role of non733 enzymatic reactions in cellular metabolism. Curr Opin Biotechnol 34, 153-161 (2015).

734 27. Breen, M.S. et al., Epistasis as the primary factor in molecular evolution.

735 Nature 490, 535-538 (2012).

736 28. de Visser, J.A., Cooper, T.F., \& Elena, S.F., The causes of epistasis. Proc Biol Sci $737 \quad 278,3617-3624(2011)$.

738 29. Harms, M.J. \& Thornton, J.W., Evolutionary biochemistry: revealing the 739 historical and physical causes of protein properties. Nat Rev Genet 14, 559-571 (2013).

740 30. Kaltenbach, M. \& Tokuriki, N., Dynamics and constraints of enzyme 741 evolution. J Exp Zool B Mol Dev Evol 322, 468-487 (2014). 
31. McCandlish, D.M. et al., The role of epistasis in protein evolution. Nature 497,

743 E1-2; discussion E2-3 (2013).

744 32. Poelwijk, F.J., Kiviet, D.J., Weinreich, D.M., \& Tans, S.J., Empirical fitness

745 landscapes reveal accessible evolutionary paths. Nature 445, 383-386 (2007).

746 33. Whitlock, M.C., Phillips, P.C., Moore, F.B.-G., \& Tonsor, S.J., Multiple Fitness

747 Peaks and Epistasis. Annu. Rev. Ecol. Syst. 26, 601-629 (1995).

748 34. Trudeau, D.L., Kaltenbach, M., \& Tawfik, D.S., On the Potential Origins of the 749 High Stability of Reconstructed Ancestral Proteins. Mol Biol Evol (2016).

750 35. Noor, S. et al., Intramolecular epistasis and the evolution of a new enzymatic 751 function. PLoS One 7, e39822 (2012).

752 36. Lozovsky, E.R. et al., Stepwise acquisition of pyrimethamine resistance in the 753 malaria parasite. Proc Natl Acad Sci U S A 106, 12025-12030 (2009).

754 37. Kiss, G. et al., Computational enzyme design. Angew Chem Int Ed Engl 52, $755 \quad 5700-5725(2013)$.

756 38. Kries, H., Blomberg, R., \& Hilvert, D., De novo enzymes by computational 757 design. Curr Opin Chem Biol 17, 221-228 (2013).

758 39. Lassila, J.K., Conformational diversity and computational enzyme design. Curr 759 Opin Chem Biol 14, 676-682 (2010).

760 40. Blomberg, R. et al., Precision is essential for efficient catalysis in an evolved 761 Kemp eliminase. Nature 503, 418-421 (2013).

762 41. Khersonsky, O. et al., Bridging the gaps in design methodologies by 763 evolutionary optimization of the stability and proficiency of designed Kemp eliminase 764 KE59. Proc Natl Acad Sci U S A 109, 10358-10363 (2012). 
765 42. Matasci, N. et al., Data access for the 1,000 Plants (1KP) project. Gigascience 3,

$76617(2014)$.

767 43. Armougom, F. et al., Expresso: automatic incorporation of structural 768 information in multiple sequence alignments using 3D-Coffee. Nucleic Acids Res 34, 769 W604-608 (2006).

770 44. Ronquist, F. \& Huelsenbeck, J.P., MrBayes 3: Bayesian phylogenetic inference 771 under mixed models. Bioinformatics 19, 1572-1574 (2003).

772 45. Cronk, Q.C., Plant evolution and development in a post-genomic context. Nat 773 Rev Genet 2, 607-619 (2001).

774 46. Ashkenazy, H. et al., FastML: a web server for probabilistic reconstruction of 775 ancestral sequences. Nucleic Acids Res 40, W580-584 (2012).

776 47. Miranda, C.L. et al., Antioxidant and prooxidant actions of prenylated and 777 nonprenylated chalcones and flavanones in vitro. J Agric Food Chem 48, 3876-3884 778 (2000).

779 48. Ashkenazy, H. et al., ConSurf 2010: calculating evolutionary conservation in 780 sequence and structure of proteins and nucleic acids. Nucleic Acids Res 38, W529-533 781 (2010).

782 49. Herman, A. \& Tawfik, D.S., Incorporating Synthetic Oligonucleotides via 783 Gene Reassembly (ISOR): a versatile tool for generating targeted libraries. Protein Eng 784 Des Sel 20, 219-226 (2007).

785 50. Zhao, H. et al., Molecular evolution by staggered extension process (StEP) in 786 vitro recombination. Nat Biotechnol 16, 258-261 (1998).

787 51. Battye, T.G. et al., iMOSFLM: a new graphical interface for diffraction-image 788 processing with MOSFLM. Acta Crystallogr D Biol Crystallogr 67, 271-281 (2011). 
789 52. Evans, P., Scaling and assessment of data quality. Acta Crystallogr D Biol 790 Crystallogr 62, 72-82 (2006).

791 53. McCoy, A.J. et al., Phaser crystallographic software. J Appl Crystallogr 40, 658$674(2007)$.

793 54. Emsley, P., Lohkamp, B., Scott, W.G., \& Cowtan, K., Features and 794 development of Coot. Acta Crystallogr D Biol Crystallogr 66, 486-501 (2010).

795 55. Adams, P.D. et al., PHENIX: a comprehensive Python-based system for 796 macromolecular structure solution. Acta Crystallogr D Biol Crystallogr 66, 213-221 797 (2010).

798 56. Goddard, T.D. \& Kneller, D.G., SPARKY 3. University of California, San 799 Francisco (2008).

800 57. Bieri, M., d'Auvergne, E.J., \& Gooley, P.R., relaxGUI: a new software for fast 801 and simple NMR relaxation data analysis and calculation of ps-ns and mus motion of 802 proteins. J Biomol NMR 50, 147-155 (2011).

803 58. Delaglio, F. et al., NMRPipe: a multidimensional spectral processing system 804 based on UNIX pipes. J Biomol NMR 6, 277-293 (1995).

805 59. Fiser, A. \& Sali, A., Modeller: generation and refinement of homology-based 806 protein structure models. Methods Enzymol 374, 461-491 (2003).

807 60. Sondergaard, C.R., Olsson, M.H., Rostkowski, M., \& Jensen, J.H., Improved 808 Treatment of Ligands and Coupling Effects in Empirical Calculation and 809 Rationalization of pKa Values. J Chem Theory Comput 7, 2284-2295 (2011).

810 61. Case, D.A. et al., AMBER 2016. University of California, San Francisco (2016).

811 62. Maier, J.A. et al., ff14SB: Improving the Accuracy of Protein Side Chain and 812 Backbone Parameters from ff99SB. J Chem Theory Comput 11, 3696-3713 (2015). 
813 63. Wang, J. et al., Development and testing of a general amber force field. $J$

814 Comput Chem 25, 1157-1174 (2004).

815 64. M. J. Frisch, G.W.T., H. B. Schlegel, G. E. Scuseria, M. A. Robb, J. R.

816 Cheeseman, G. Scalmani, V. Barone, G. A. Petersson, H. Nakatsuji, X. Li, M. Caricato,

817 A. Marenich, J. Bloino, B. G. Janesko, R. Gomperts, B. Mennucci, H. P. Hratchian, J.

818 V. Ortiz, A. F. Izmaylov, J. L. Sonnenberg, D. Williams-Young, F. Ding, F. Lipparini, F.

819 Egidi, J. Goings, B. Peng, A. Petrone, T. Henderson, D. Ranasinghe, V. G. Zakrzewski,

820 J. Gao, N. Rega, G. Zheng, W. Liang, M. Hada, M. Ehara, K. Toyota, R. Fukuda, J.

821 Hasegawa, M. Ishida, T. Nakajima, Y. Honda, O. Kitao, H. Nakai, T. Vreven, K.

822 Throssell, J. A. Montgomery, Jr., J. E. Peralta, F. Ogliaro, M. Bearpark, J. J. Heyd, E.

823 Brothers, K. N. Kudin, V. N. Staroverov, T. Keith, R. Kobayashi, J. Normand, K.

824 Raghavachari, A. Rendell, J. C. Burant, S. S. Iyengar, J. Tomasi, M. Cossi, J. M. Millam,

825 M. Klene, C. Adamo, R. Cammi, J. W. Ochterski, R. L. Martin, K. Morokuma, O.

826 Farkas, J. B. Foresman, D. J. Fox, Gaussian 09, Revision D.01. Gaussian, Inc.,

827 Wallingford CT (2016).

828 65. Cieplak, P., Cornell, W.D., Bayly, C., \& Kollman, P.A., Application of the 829 multimolecule and multiconformational RESP methodology to biopolymers: Charge 830 derivation for DNA, RNA, and proteins. J Comput Chem 16, 1357-1377 (1995).

831 66. Jorgensen, W.L. et al., Comparison of simple potential functions for 832 simulating liquid water. The Journal of Chemical Physics 79, 926-935 (1983).

833 67. Berendsen, H.J.C. et al., Molecular dynamics with coupling to an external bath. 834 Journal of Chemical Physics 81, 3684-3690 (1984). 
835 68. Ryckaert, J.-P., Ciccotti, G., \& Berendsen, H.J.C., Numerical integration of the

836 Cartesian Equations of Motion of a System with Constraints: Molecular Dynamics of

837 n-Alkanes. Journal of Computational Physics 23, 327-341 (1977).

838 69. Feller, S.E., Zhang, Y., Pastor, R.W., \& Brooks, B.R., Constant pressure

839 molecular dynamics simulation: The Langevin piston method. The Journal of

840 Chemical Physics 103, 4613-4621 (1995).

841 70. Darden, T., York, D., \& Pederson, L., Particle Mesh Ewald: An N•log(N)

842 Method for Ewald Sums in Large Systems. Journal of Chemical Physics 98,

843 10089-10092 (1993).

844 71. Humphrey, W., Dalke, A., \& Schulten, K., VMD: visual molecular dynamics. J

845 Mol Graph 14, 33-38, 27-38 (1996).

846 72. McGibbon, R.T. et al., MDTraj: A Modern Open Library for the Analysis of

847 Molecular Dynamics Trajectories. Biophys J 109, 1528-1532 (2015).

848 73. Pettersen, E.F. et al., UCSF Chimera--a visualization system for exploratory

849 research and analysis. J Comput Chem 25, 1605-1612 (2004). 


\section{Figure 1}

a<smiles>O=C(/C=C/c1ccc(O)cc1)c1c(O)cc(O)cc1O</smiles>

Chalconaringenin<smiles>CC(C)C1CC(=O)c2c(O)c[13c](O)cc2O1</smiles>

b

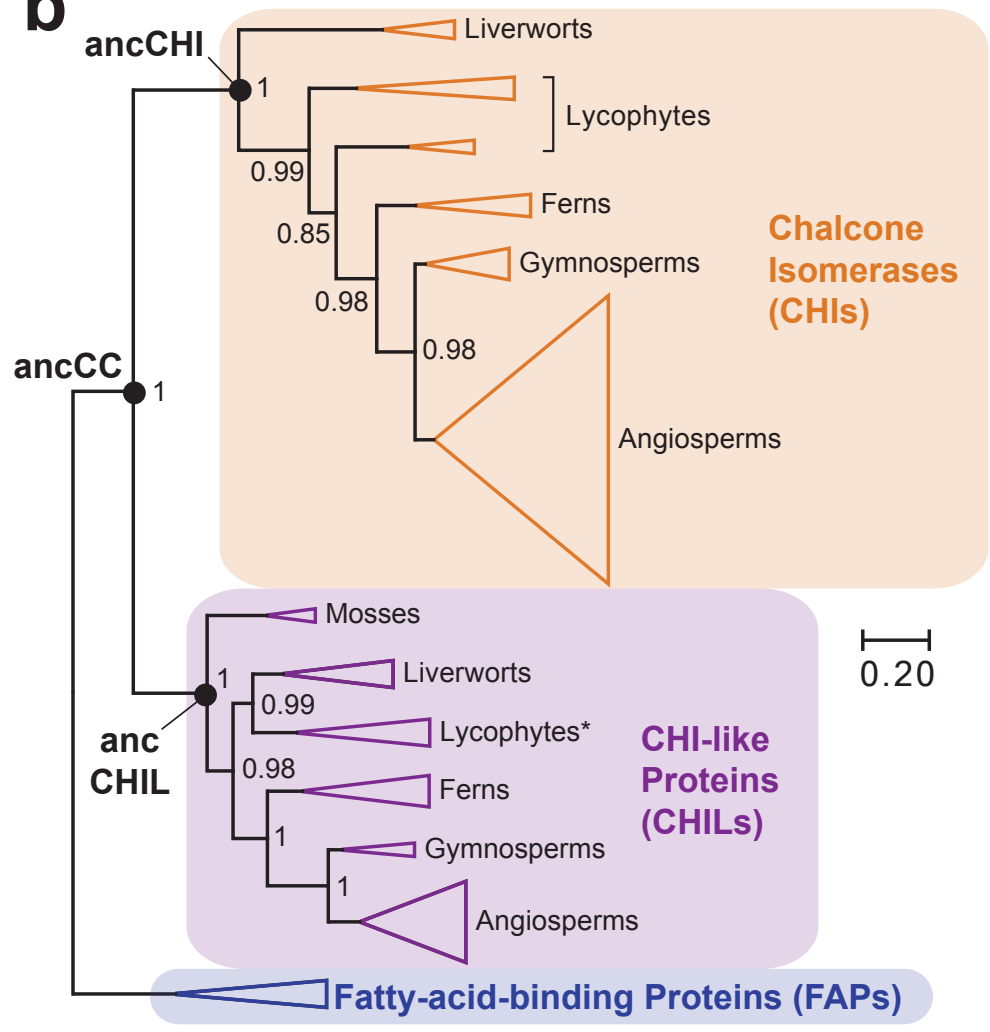

Figure 1. The $\mathrm{CHI}$ protein family. (a) $\mathrm{CHI}$ catalyzes the intramolecular Michael addition of chalconaringenin (2',4,4',6'-tetrahydroxychalcone or (2E)-3-(4-hydroxyphenyl)-1-(2,4,6-trihydroxyphenyl)prop-2en-1-one) to yield the flavonoid naringenin (5,7-Dihydroxy-2-(4-hydroxyphenyl)chroman-4-one). (b) Cartoon representation of CHI's phylogenetic tree. Posterior probabilities and the inferred ancestral nodes are annotated. The complete phylogenetic tree is shown in Supplementary Fig. $\mathbf{2}$ and the alignment used to generate it is given in Supplementary Fig. 1 and Supplementary Data Set 1. 


\section{Figure 2}

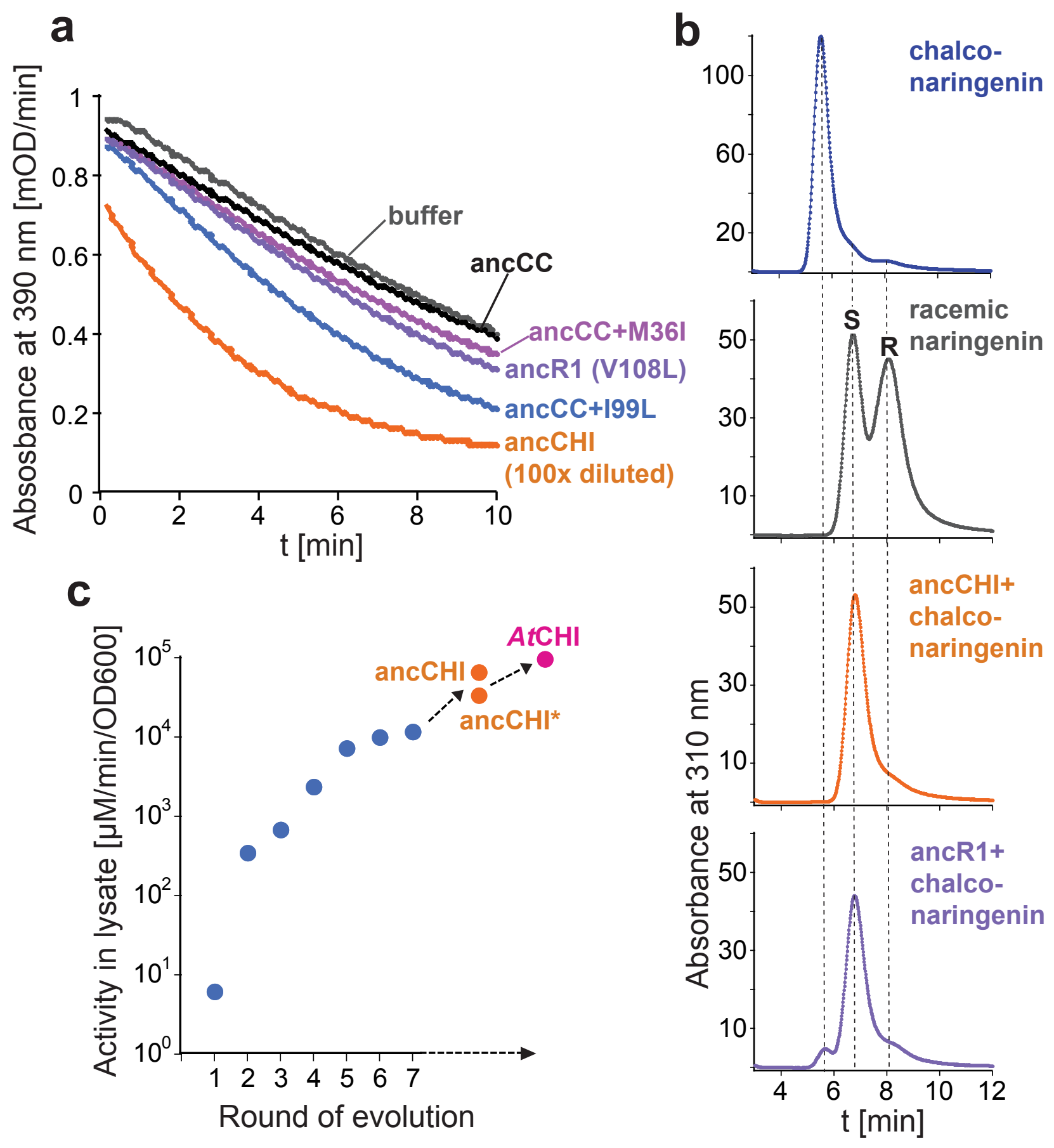

Figure 2. Isomerase activity of the inferred ancestors and evolutionary intermediates. (a) Kinetics of naringenin formation indicated by the decrease in 390 $\mathrm{nm}$ absorbance. While ancCHI is a highly active isomerase, no turnover of naringenin chalcone above background (buffer) could be detected for ancCC at $\geq 100$-fold higher protein concentrations. The three founder mutations induce a low yet reproducible rate enhancement. (b) Steroselectivity of $\mathrm{CHI}$ variants. Like all characterized extant enzymes, all evolutionary intermediates produce (S)-naringenin. (c) The evolutionary trajectory leading from ancCC to ancCHI*. The underlying mutatons are shown in Supplementary Table 8. The $Y$-axis denotes isomerase activity under the screening conditions (i.e., in clarified lysates of the E. coli cells in which these variants were expressed). AncCHI and $\mathrm{AtCHI}$ are shown for comparison. Specific activities measured with purified proteins and kinetic parameters are provided in Supplementary Tables $\mathbf{4}$ and 9. 


\section{Figure 3}

a

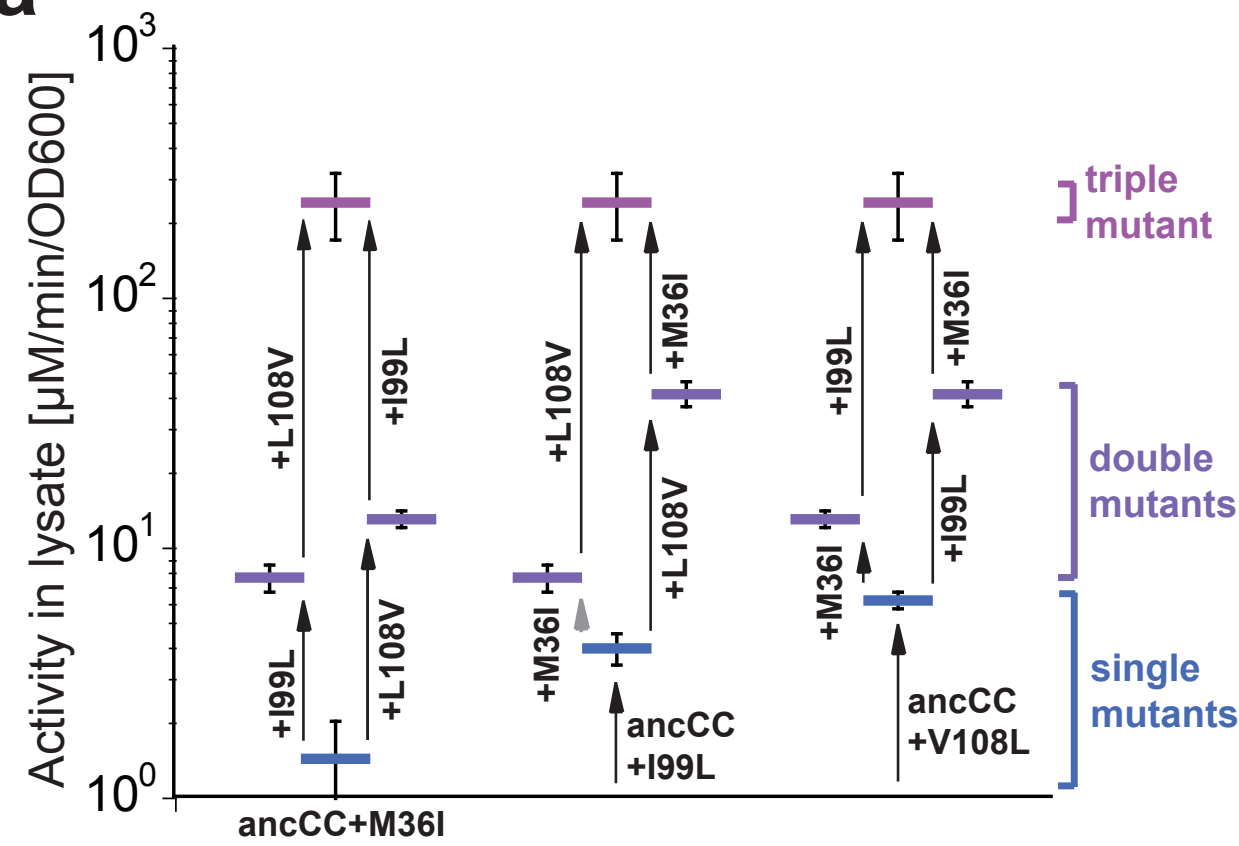

b

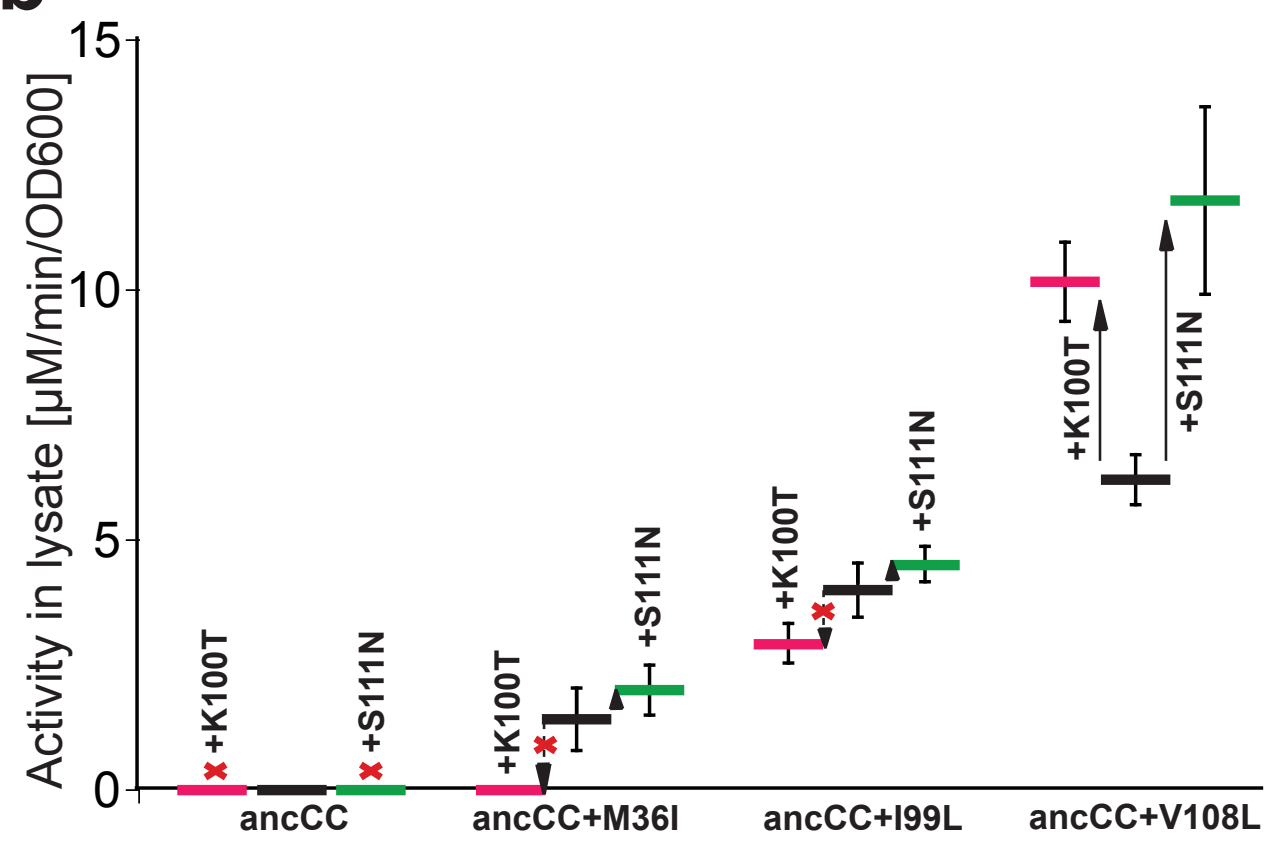

Figure 3. Additivity versus epistasis along CHI's evolution. (a) The three founder mutations (L108V, 199L and M36I) can be combined in any order in an uphill trajectory. (b) Two additional mutations that appeared later in the trajectory, K100T and S111N, only develop their effect if L108V is present. Initial rates were determined in clarified cell lysates. Specific activities obtained with purified protein are provided in Supplementary Tables 4 and 9. 


\section{Figure 4}

a

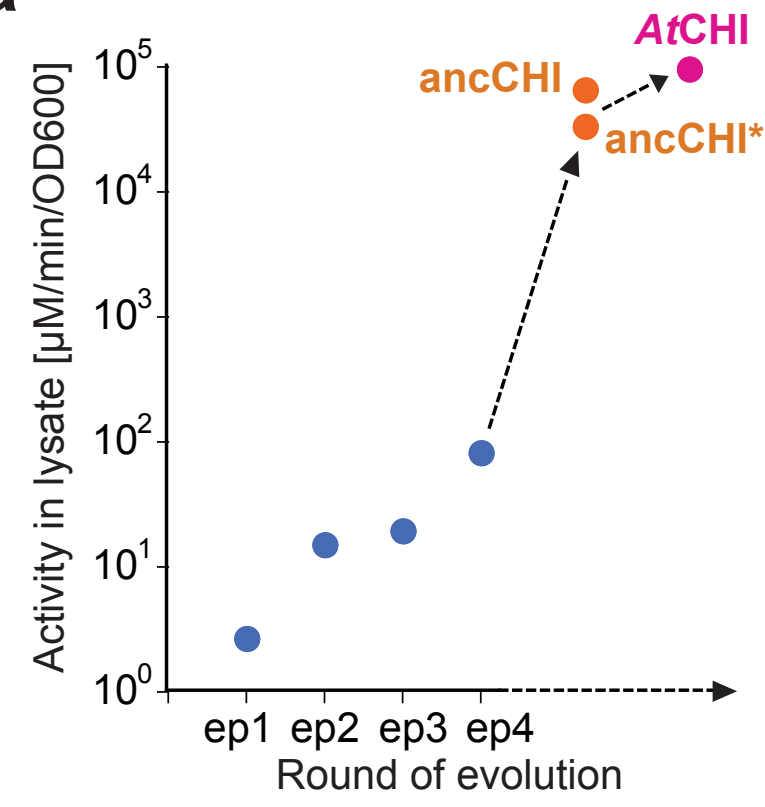

b

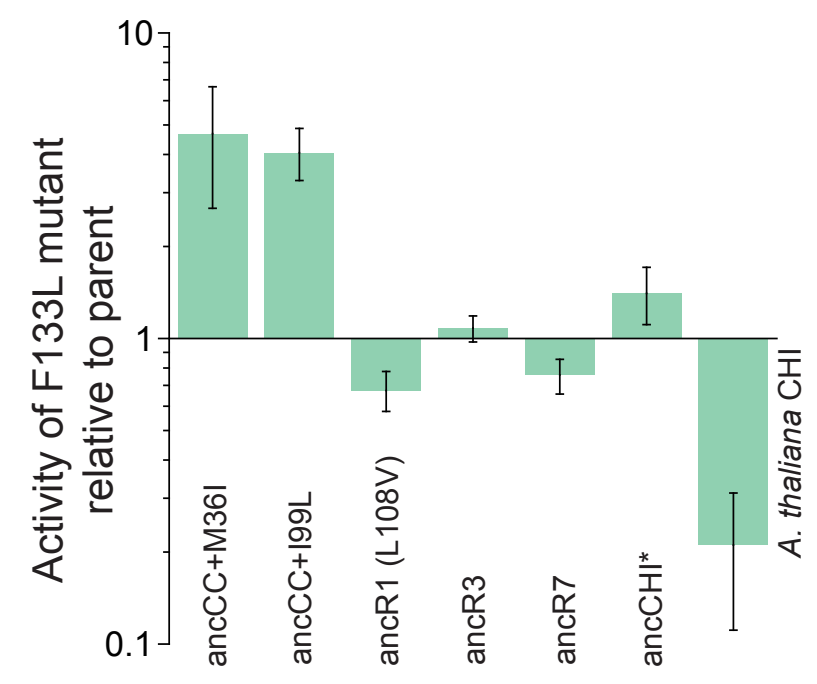

Figure 4. The alternative random mutagenesis trajectory. (a) Development of isomerase activity along the trajectory (as in Fig. 2c). The underlying mutations are shown in Supplementary Table 13. (b) The effect of F133L, the founder mutation of the alternative trajectory, changes depending on the genetic background. Shown is the ratio of activities of different variants with and without the F133L mutation. Specific activities and kinetic parameters measured with purified protein are provided in Supplementary Tables 4 and 9 . F133 corresponds to F146 in AtCHI. 
a

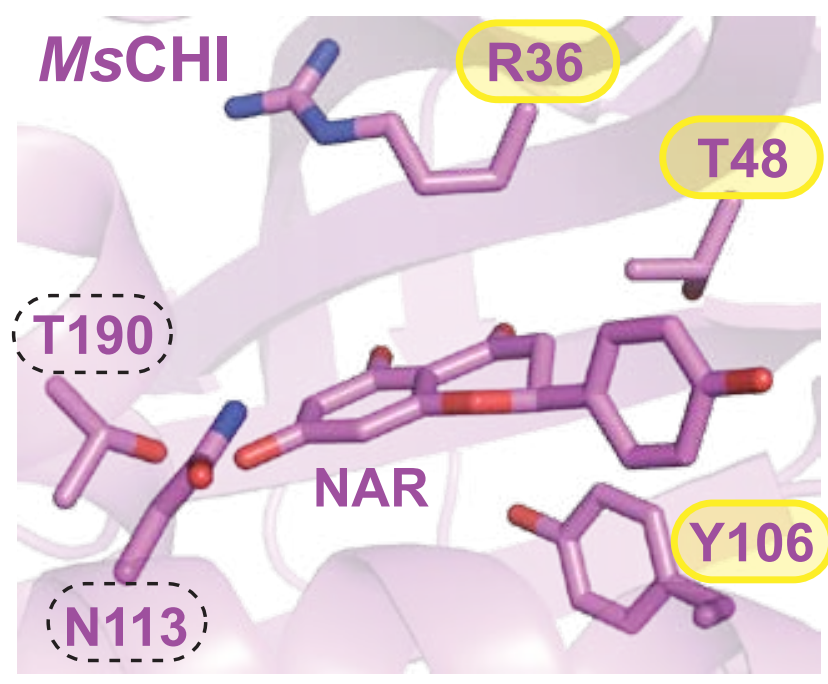

b

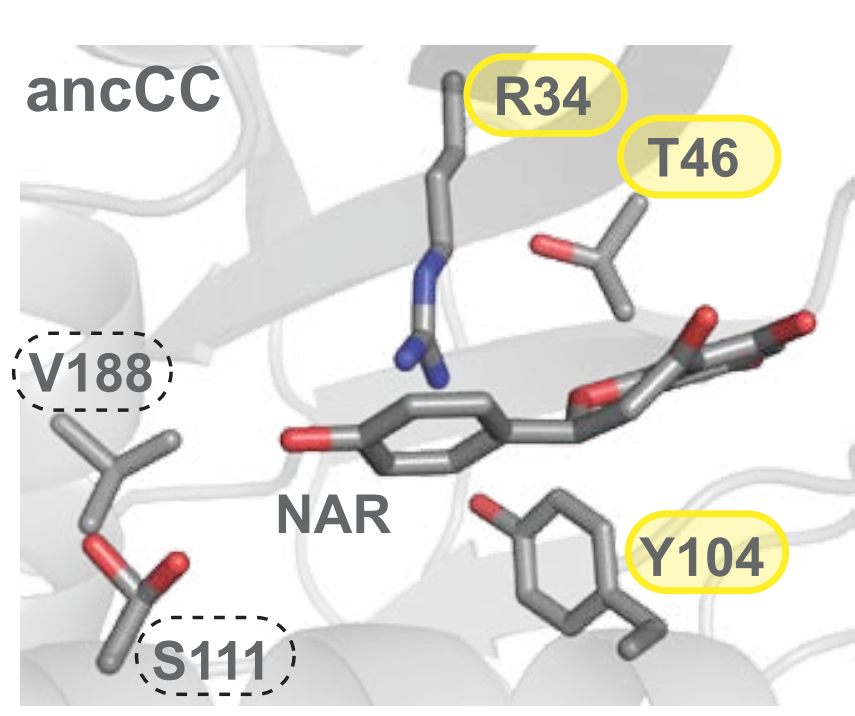

C

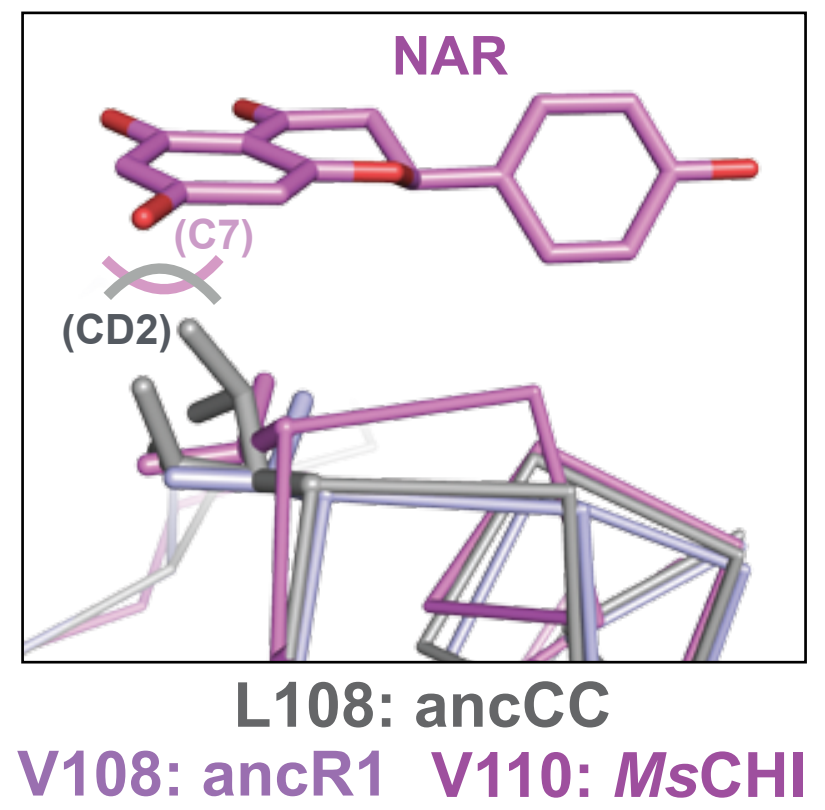

d
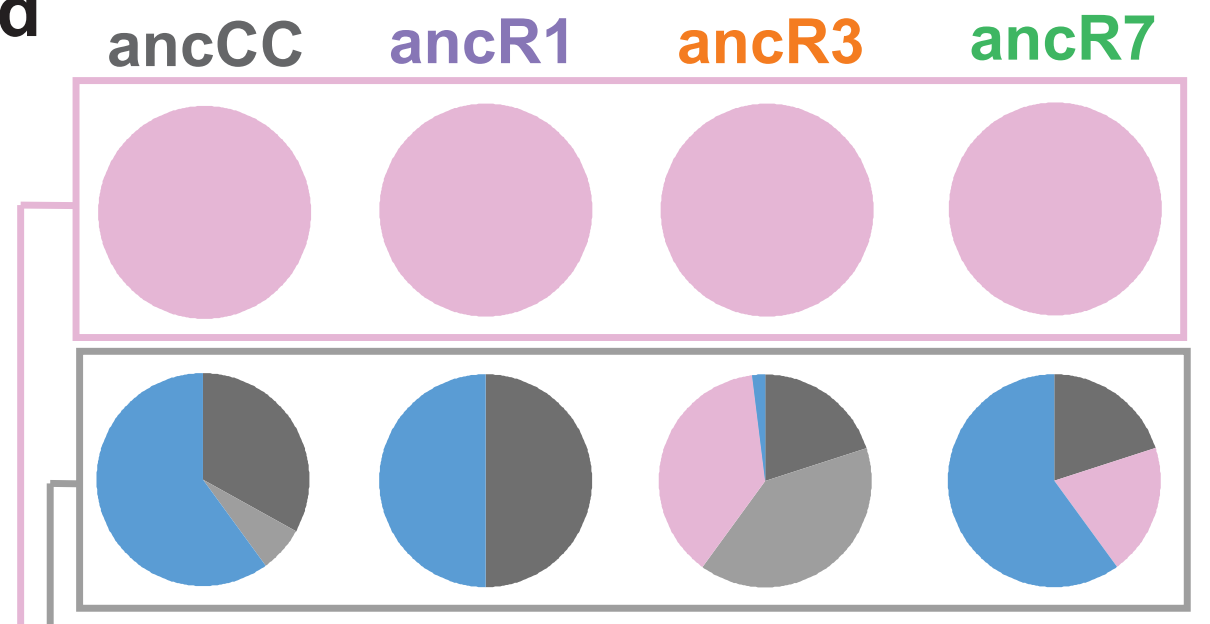

Initial binding mode:

CHI-like, productive

Initial binding mode: ancCC-like, non-productive
Final 50 ns binding mode:

CHI-like, productive

ancCC-like, non-productive

n other binding modes

\section{dissociated}

Figure 5. Structural changes over the evolution. (a) Crystal structure of $\mathrm{MsCHI}$ [19] with (S)-naringenin (NAR) bound in the CHI-like mode. Selected active-site residues are labelled. (b) Crystal structure of ancCC with naringenin bound in the ancCC-like mode. The three key residues are already present (yellow circles). (c) The structural alignment of ancCC and ancR1 with $\mathrm{MsCHI}$ with (S)-naringenin bound (Ca alignment with PyMOL, RMSD = 1.14 ̊̊) reveals a likely steric clash between (S)-naringenin and the side chain of L108 in ancCC (distance CD2 to C7 2 $\AA$ A), suggesting that the $\mathrm{CHI}$-like binding mode is incompatible with ancCC. AncR1 harbors an activating substitution, L108V, which alleviates the steric clash by reducing the length of the side chain by a methylene moiety, thus increasing active site volume. (d) Percentage of simulation time in which the substrate assumes a particular binding mode in ancCC, ancR1, ancR3, and ancR7 after initially being placed in either the productive (top panel) or non-productive mode (bottom panel). Each variant was simulated in five replicas (100 ns each). The last $50 \mathrm{~ns}$ of each simulation were combined and RMSD-based clustering using the average linkage algorithm was applied to calculate the fraction of time in which the substrate resides in the productive, non-productive, or other binding modes (illustrated in Supplementary Fig. 11) or dissociates from the active site. Note that productive and non-productive modes include structures in which the substrate is not ideally aligned with either of the two modes, with some RMSD fluctuations still observed, but retaining the proper orientation and being fully bound in the active site. Additional simulations performed with ancCHI and AtCHI are shown in Supplementary Fig.s 9-10. 

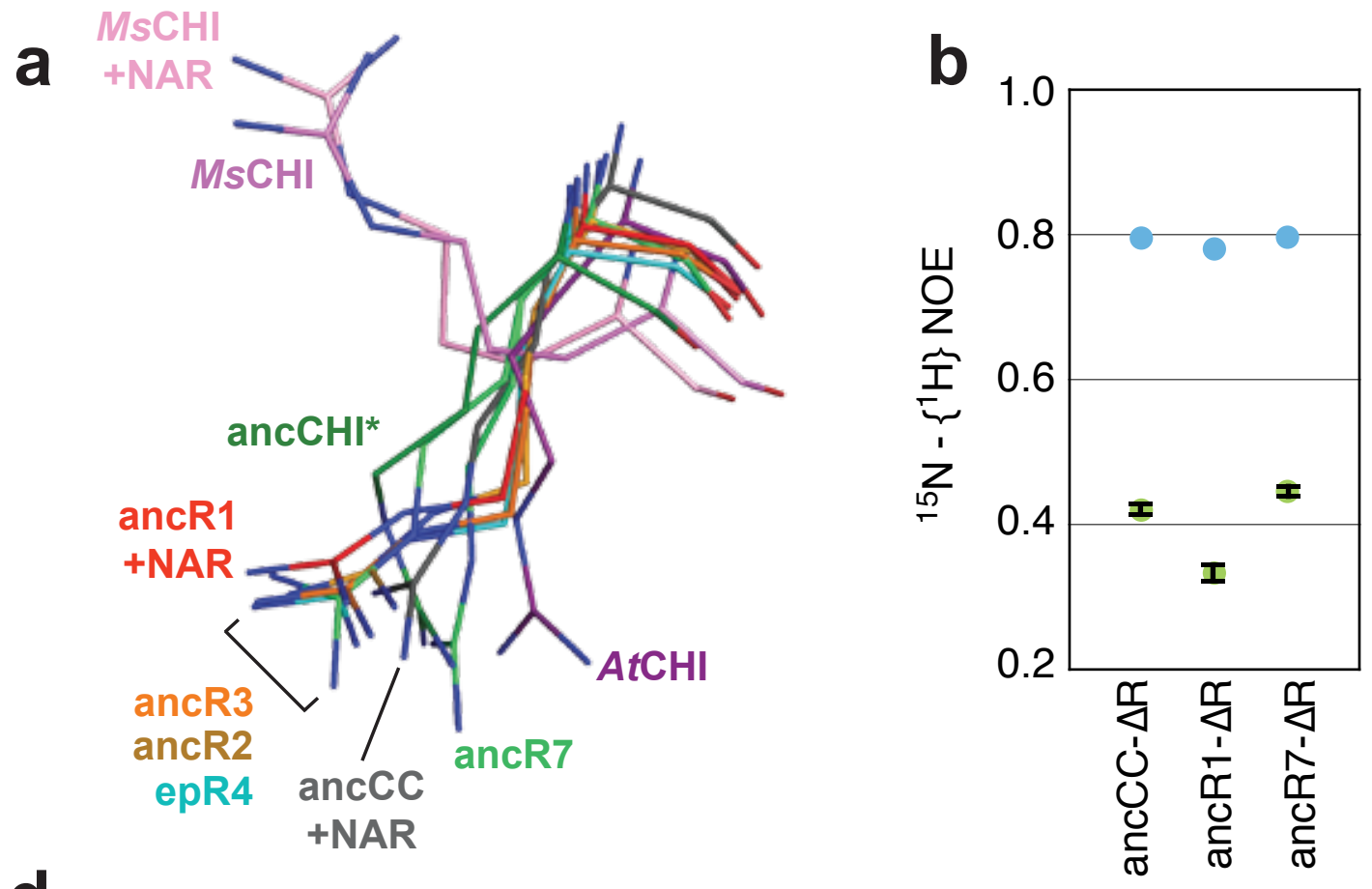

Figure 6
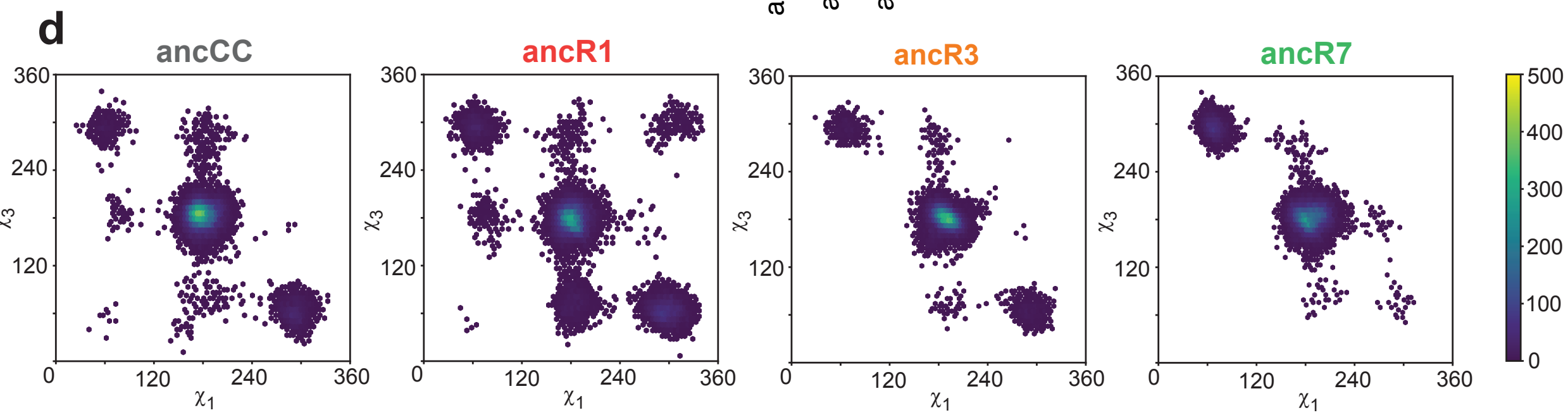

Figure 6. The conformational ensemble of the catalytic Arginine changes over the evolution. (a) The structural alignment of different crystal structures reveals that the conformation of R34 varies widely. The presence of (S)-naringenin (NAR) does not significantly influence this conformation. The structure of AtCHI (PDB: 4doi) was reported in [7], the structure of MsCHI

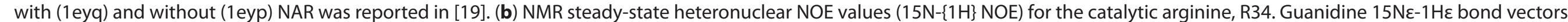
(green), and average values for backbone amide $15 \mathrm{~N}-1 \mathrm{H}$ bond vectors are shown for ancCC-, ancR1-, and ancR7- $\triangle \mathrm{R}$ (blue, using resonance peaks downfield of 8.5 ppm). Low $15 \mathrm{~N}-\{1 \mathrm{H}\} \mathrm{NOE}$ values indicate mobility on the pico-nanosecond timescale relative to overall molecular tumbling. These experiments reveal that the mobility of the arginine side chain first increases, ther decreases at key points in the evolutionary trajectory but remains mobile on the pico-nanosecond timescale for the most active variant, ancR7- $\Delta R$. Average values of triplicate experiments are shown and error bars are standard deviations. (c) The distinct HSQC signals of the arginine side chain are indicative of a change in its chemical environment over the evolution. (d) Correlated distribution of the $x 1$ and $x 3$ dihedral angle values sampled by the Arg34 side chain in ancCC, ancR1, ancR3, and ancR7. Two-dimensional histograms were calculated from the last $50 \mathrm{~ns}$ of the simulation of each variant, repeated in five independent replicas, i.e. $250 \mathrm{~ns}$ of sampling in each case (or four replicas/200 ns in the case of ancR3), and the angle values are reported in degrees. 